Article

\title{
Optimal Allocation and Planning of Distributed Power Generation Resources in a Smart Distribution Network Using the Manta Ray Foraging Optimization Algorithm
}

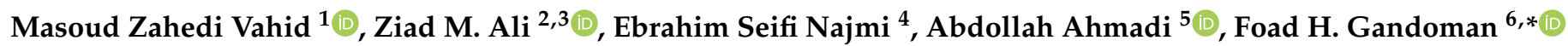 \\ and Shady H. E. Abdel Aleem ${ }^{7}$ (1) \\ 1 Department of Electrical and Computer Engineering, University of Sistan and Baluchestan, \\ Zahedan 9816745785, Iran; mzahedivahid@pgs.usb.ac.ir \\ 2 Electrical Engineering Department, Aswan faculty of Engineering, Aswan University, Aswan 81542, Egypt; \\ dr.ziad.elhalwany@aswu.edu.eg \\ 3 Electrical Engineering Department, College of Engineering at Wadi Addawaser, Prince Sattam bin Abdulaziz \\ University, Wadi Addawaser 11991, Saudi Arabia \\ 4 Roshdieh Higher Institue of Education, Tabriz 5166616471, Iran; ebrahimseifinajmi@gmail.com \\ 5 School of Electrical Engineering and Telecommunications, The University of New South Wales, \\ Sydney 2052, Australia; ahmadi.abdollah.janah@gmail.com \\ 6 Research Group MOBI-Mobility, Logistics, and Automotive Technology Research Centre, \\ Vrije Universiteit Brussel, 1050 Brussels, Belgium \\ 7 Department of Electrical Engineering and Electronics, Valley Higher Institute of Engineering and Technology, \\ Science Valley Academy, Al-Qalyubia 44971, Egypt; engyshady@ieee.org \\ check for \\ updates \\ * Correspondence: Foad.heidari.Gandoman@VUB.be
}

Citation: Zahedi Vahid, M.; Ali, Z.M.; Seifi Najmi, E.; Ahmadi, A.; Gandoman, F.H.; Aleem, S.H.E.A. Optimal Allocation and Planning of Distributed Power Generation Resources in a Smart Distribution Network Using the Manta Ray Foraging Optimization Algorithm. Energies 2021, 14, 4856. https:// doi.org/10.3390/en14164856

Academic Editor: Michela Robba

Received: 21 June 2021

Accepted: 4 August 2021

Published: 9 August 2021

Publisher's Note: MDPI stays neutral with regard to jurisdictional claims in published maps and institutional affiliations.

Copyright: (c) 2021 by the authors. Licensee MDPI, Basel, Switzerland. This article is an open access article distributed under the terms and conditions of the Creative Commons Attribution (CC BY) license (https:// creativecommons.org/licenses/by/ $4.0 /)$.

\begin{abstract}
In this study, optimal allocation and planning of power generation resources as distributed generation with scheduling capability (DGSC) is presented in a smart environment with the objective of reducing losses and considering enhancing the voltage profile is performed using the manta ray foraging optimization (MRFO) algorithm. The DGSC refers to resources that can be scheduled and their generation can be determined based on network requirements. The main purpose of this study is to schedule and intelligent distribution of the DGSCs in the smart and conventional distribution network to enhance its operation. First, allocation of the DGSCs is done based on weighted coefficient method and then the scheduling of the DGSCs is implemented in the 69-bus distribution network. In this study, the effect of smart network by providing real load in minimizing daily energy losses is compared with the network includes conventional load (estimated load as three-level load). The simulation results cleared that optimal allocation and planning of the DGSCs can be improved the distribution network operation with reducing the power losses and also enhancing the voltage profile. The obtained results confirmed superiority of the MRFO compared with well-known particle swarm optimization (PSO) in the DGSCs allocation. The results also showed that increasing the number of DGSCs reduces more losses and improves more the network voltage profile. The achieved results demonstrated that the energy loss in smart network is less than the network with conventional load. In other words, any error in forecasting load demand leads to non-optimal operating point and more energy losses.
\end{abstract}

Keywords: smart distribution network; distributed generation with scheduling capability; power generation resources; manta ray foraging optimization algorithm

\section{Introduction}

In the power system, a significant part of power losses is related to the distribution sector [1]. The most common approaches to minimize losses in distribution networks include reconfiguration [2], capacitance allocation [3-5] and distributed generation (DG) allocation [6,7]. DG allocation is one of the most convenient methods because of the advantages of reducing losses, reducing voltage deviations, improving reliability, and selling 
power. Improper allocation and irrational and non-optimal planning of these resources weaken the network performance [8,9]. Optimal DG placement can be applied to all types of DGs and implemented in the design phase with the help of general network information, mainly related to the system worst conditions (e.g., peak load condition). While DGs scheduling can only be implemented for dispatchable or schedulable DGs, such as fuel cells, micro-turbines, and diesel generators. It is worth noting here that DGs can be placed in two major categories, schedulable and non-schedulable. The schedulable DGs such as micro-turbines, gas engines, and fuel cells are sourcing whose output can be controlled by operators. The non-schedulable are such that their output is uncontrollable. Wind turbines and photovoltaic systems are two well-known non-schedulable types. The optimal performance of distributed generation with scheduling capability (DGSC) depends on the network conditions and will change as these conditions change. Therefore, the best operational point of DGSC should be updated when network conditions change. More accurate and accurate information on the network conditions will lead to better performance of the DGSCs [10-12]. The network load is one of the parameters that are constantly changing in the network and having more accurate information about it improves the performance of DGs.

Few studies have been done on the optimal planning of the DGSCs in the distribution network, especially smart distribution networks [13,14]. Many methodologies have been applied for allocation of the DGSCs in the network. Some of these methods are mentioned first and then the studies in the field of smart grids are discussed. The location of wind and photovoltaic resources in the network is presented and aimed with reducing losses and improving voltage stability via particle swarm optimization (PSO) algorithm in [15]. In [16], optimal allocation of renewable resources via evolutionary programming (EP) is presented in the network with objective of loss reduction and enhancing the voltage profile. In [17], a high-efficiency algorithm is suggested using the firefly algorithm (FA) to find the site and capacity of DGs in an unbalanced network with objective for loss reduction. In [18], network reconfiguration and allocation of DGs is performed for reducing loss minimization and enhancing the voltage profiles via plant growth algorithm (PGA). In [19], network reconfiguration with allocation of DGs is developed for losses and voltage deviations minimization with cuckoo search algorithm (CSA). In [20], allocation of DGs is presented for loss reduction and reliability enhancement with dynamic programming. In [21], optimal allocation of DGs for minimizing the loss of energy have been done in a smart grid but effect of forecasting error on load profiles has not been investigated. In [22], the optimal performance of DGs to minimize energy losses in smart network is compared with conventional network. Also, in [22], the effect of three-level load on the network performance is not evaluated in the smart environment. In [23], optimal application of DGSCs is presented with the objective of minimizing energy losses using the PSO. The purpose of this study is to propose a DG planning model that allows operator to optimally plan different types of DG, to reduce the loss of energy. In [24], the location of DGs, automatic voltage control, types of generators and modes of operation of DGs is investigated on the smart network. The results showed that if DG is exploited in current and voltage control modes, losses are significantly reduced. Using the proposed method, the losses can be reduced by selecting different DG technologies. In [25], optimal planning of the DGSCs with aim of minimizing energy losses in the smart and traditional networks using the teaching-learning optimization (TLO) method has been investigated but the voltage profile enhancement is not considered in network performance analysis. In [26], the advantages of smart network and distribution management system are presented on the losses, reduction of fixed costs, network development costs and power quality improvement. The results showed that the suggested methodology is a suitable approach to improve the performance of the network. In [27], the effect of smart networks is performed on reducing the cost of network losses. In this study, commercial locations that have the potential for high losses are determined thus the cost of electricity declines and great benefit is obtained from reducing losses. In [28], interconnection of energy resources in smart 
networks is developed using the evolutionary algorithm and optimal location of DGSCs with the ability to inject reactive power is optimally determined by the GA to achieve the appropriate solution.

As it is found from the literature review, most of the studies for allocation and improving the performance of DGs have been done considering the peak load and for the worst network conditions. To plan the DGSCs in the distribution network, selecting a load with multi-level (estimated load model in conventional network) is desirable and operational point must be extracted with the aim of minimizing energy losses. This multi-level load model can be accurately determined in smart networks based on measuring devices. Therefore, a more accurate load model can lead to better scheduling of the network based on DGSCs [27,28]. It is necessary to evaluate the effect of using smart load in improving the network performance compared to conventional network loads in reducing energy losses. So, in this study, the effect of considering smart load in improving the distribution network performance is compared with conventional network load with considering load forecasting error. It is also necessary to use a powerful optimization method with high optimization accuracy to achieve a global solution to determine the best location to install the DGSCs so that accurate distribution network planning can be performed based on it. Why, given the introduction of several optimization algorithms in the past, it is still necessary to provide new algorithms to solve the optimization problem? The answer to this question is in the No Free Lunch optimization theory [29,30], which states that no algorithm has the best capability for all optimization problems solving. Therefore, using an algorithm with optimal optimization capability is one of the goals to solve the problem presented in this study.

In this paper, optimal allocation, and planning of power generation resources as DGSCs is presented in distribution network with aim of minimizing the losses considering enhancing the network voltage profile using manta ray foraging optimization (MRFO) algorithm [31,32] as a 24-h study. Superiority of the MRFO method in DGSCs allocation is compared with PSO method. The effect of using smart load compared to the estimated three-level load is investigated in reducing network energy losses. Accordingly, optimal management of DGSCs distribution in the smart network is implemented with objective of loss of energy minimization. In this research effect of smart and conventional networks load is compared in view of energy losses reduction via the MRFO. The suggested methodology is implemented on 69-bus distribution network and the effect of load forecasting error is evaluated on improving the network performance.

Research contributions are provided below:

- Optimal allocation and planning of DGSCs in distribution network

- Evaluating the effect of smart and estimated load on energy losses

- Undermining network performance due to load forecasting error

- More net saving with by moving networks towards smartening

- Superiority of the MRFO compared to PSO

In Section 2, problem formulation includes objective function of allocation and planning of DGSCs in the distribution network and also constraints are presented. The load models of the network considering conventional and smart load are explained in Section 3. The proposed optimization method is named manta ray foraging method and its application steps to solve the problem are described in Section 4. The findings results are given in Section 5. Finally, in Section 6 main findings are concluded.

\section{Problem Formulation}

In this study, the effect of smart network with the optimal allocation of DGSCs is evaluated with objective of losses reduction and enhancing the voltage profile via the MRFO. The objective function is presented along with the constraints. Load models for conventional and smart distribution networks are also presented in different cases. 


\subsection{Optimal Positioning Objective Function}

The DGSCs reduce line power flow and reduce active power losses by installation at the load consumption site. The non-optimal allocation in the network causes poor performance of distribution networks. So, to improve the network performance based on the DGSCs, the installation location should be determined optimally $[1,6,11]$. The total objective function considering the goals of minimizing active power losses and enhancing the voltage profile (minimization of the voltage deviations) is defined as follows:

$$
\begin{gathered}
\min \text { OF }=\text { Power_Loss\&Voltage_Deviation }= \\
P_{\text {Loss }}^{\text {total }} \& V_{D}^{\text {total }}(x, \text { Load }) \\
\text { S.T. } g(x)=0 \\
h(x) \leq 0
\end{gathered}
$$

where, $P_{\text {Loss }}^{\text {total }}$ and $V_{D}^{\text {total }}$ are the total amounts of losses and voltage deviations of the network, considering constraints of equality $(g(x)=0)$ and inequality $(h(x) \leq 0) . x$ indicates problem variables such as location and capacity of the DGSCs and Load refers to the cases defined for the network load.

\subsubsection{Power Losses}

The amount of active power loss by passing current through network lines is obtained as follows [1,6]:

$$
P_{\text {Loss }}=\sum_{k=1}^{N_{L}} R_{k}\left(\frac{V_{i}-V_{j}}{R_{k}+j X_{k}}\right)^{2}
$$

where $V_{i}$ and $V_{j}$ are the voltages of the buses $i$ and $j$, respectively, $R_{k}$ and $X_{k}$ refer to the ohmic resistance and reactance of line $k$ and $N_{L}$ is the total line number.

\subsubsection{Voltage Profile}

Another important objective in the network operation is to minimize the voltage deviations, which is also named as enhancing the voltage profile. The sum voltage deviations is presented by $[1,6,33]$ :

$$
\begin{gathered}
V_{D}=\sqrt{\frac{1}{N_{b u s}} \times \sum_{i=1}^{N_{\text {bus }}}\left(V_{i}-V_{p}\right)^{2}} \\
V_{p}=\frac{1}{N_{b u s}} \times \sum_{i=1}^{n_{\text {bus }}} V_{i}
\end{gathered}
$$

where, $N_{b u s}$ refers to the bus numbers and $V_{p}$ is the average voltage of the buses.

\subsection{Objective Function of Optimal Planning}

Distribution of the DGSCs is performed optimally according to the general conditions of the network and in planning of the DGSCs, generation of these resources are managed according to the conditions of the network. The objective function of the planning is defined by $[1,6]$ :

$$
\begin{gathered}
\min \text { OF =Energy_Loss }=\sum_{t}^{T} P_{\text {loss }, t}^{\text {total }}\left(x_{t}, \text { Load }_{t}\right) \times t \\
\text { S.T. } g\left(x_{t}\right)=0 \\
h\left(x_{t}\right) \leq 0
\end{gathered}
$$

where $T$ indicates the study period as $24 \mathrm{~h}$. 


\subsection{Problem Constraints}

The following constraints must be met in solving the problem $[1,6]$ :

- Power capacity

The amount of DG generation in planning should not exceed the allowable amount:

$$
P_{D G S C}<P_{D G S C}^{\max }
$$

where, $P_{D G S C}^{\max }$ is maximum power of the DGSC.

- $\quad$ Limit thermal limits

The amount of current passing through the network lines should not exceed the maximum tolerable amount:

$$
F_{i}<\text { Limit }_{i}
$$

where, Limit $_{i}$ is maximum allowable current of line $i$.

- Voltage constraint

The voltage value of each network bus must be placed the allowable values:

$$
V_{i}^{\min }<V_{i}<V_{i}^{\max }
$$

where, $V_{i}^{\min }$ and $V_{i}^{\max }$ are the lower and upper limits of the bus voltage of the network.

In the search space, the optimization program selects the optimization variables, i.e., installation location and capacity of the DGSC for each population of the algorithm. By randomly determining the variables, it then checks the mentioned operating constraints. Thus, the allowable voltage range of the buses and the maximum current passing through the network lines due to placing the DGSC in the selected location with the capacity installed by the optimization program must be satisfied. Then the value of the objective function resulting from these selected variables is calculated. If the operating conditions are not met, this solution is infeasible and will be discarded. Then, the optimization program in the search space selects the other random variables, randomly. Therefore, the optimization program randomly examines the constraints after determining the variables.

\subsection{Multi-Objective Optimization}

In this research, the distribution of scattered products in the distribution network has been done with the aim of minimizing the losses and voltage deviations with weighted coefficient method [1]. The total objective function $(O F)$ with normalization of each part of the OF including losses and voltage deviations is defined by:

$$
\begin{gathered}
\min \text { imize } O F=w_{\text {Loss }}\left(\frac{P_{\text {Loss }}}{P_{\text {Loss }}^{\max }}\right)+w_{\text {Voltage }}\left(\frac{V_{D}}{V_{D}^{\max }}\right) \\
\text { s.t. } g(x)=0, h(x) \leq 0 \\
w_{\text {Loss }}+w_{\text {Voltage }}=1
\end{gathered}
$$

where, $P_{\text {Loss }}^{\max }$ and $V_{D}^{\max }$ are the maximum values of losses and voltage deviations, $w_{\text {Loss }}$ and $w_{\text {Voltage }}$ are weighted coefficients related to functions of losses and voltage deviations, respectively. The $w_{\text {Loss }}$ and $w_{\text {Voltage }}$ weight coefficients of each of the objectives of losses and voltage deviations in the multi-objective function (Equation (9)) are based on the weight coefficients method. These coefficients are presented to normalize the overall objective function. Here and in many articles, these coefficients are determined based on trial-and-error methods and user experience. However, in this study, in addition to the trialand-error method, we also used the optimization program to optimize these coefficients, the approximate value of each to obtain the best solution is 0.5 . 


\section{Load Model of the Network}

Most studies to locate and improve the performance of DGs have been done considering a load level, i.e., peak load, for the worst network conditions. In these conditions, the operational point determined in the network under changing network conditions including load changes weakens the network performance. Therefore, in case of network load changes, the operational point must be determined optimally. To better plan of DGSCs power distribution in the network, it is logical to choose a multi-level load model based on the estimated load model in a conventional network. Moreover, in smart network based on measuring devices, more accurate load profile can be achieved so that instantaneous changes in load demand can be monitored. Therefore, a more accurate model of load demand can be effective in improving the network performance $[25,26]$.

In this study, six types of load models are considered. The first case represents the real load extracted from the smart network and the other five types show the load of conventional networks based on the estimated three-level load model. Different models of the load are defined as follows:

Case (1) The first case is a 24-h load extracted from the smart network based on smart measuring devices (Figure 1).

Case (2) In this case (Figure 1) load of each level ( $8 \mathrm{~h})$ is equal to average of the smart load demand (accurate forecast).

Case (3) In this case, the load value of each level is considered equal to actual peak load value at that level and is shown in Figure 2 (correct forecasting with covering the worst load condition). Case 3 is more logical than the Case 2.

Case (4) The estimated demand is 10\% more than the three-level demand model in case 3 (Case 4 has a forecasting error).

Case (5) The estimated load is 10\% less than in the three-level load demand in case 3. (Case 5 has a forecasting error).

Case (6) This case is a combination of different modes so that in the first level the load forecast is equal to $10 \%$ less than the lowest demand value, in the second level the load forecast is equal to the peak load value and in the third level the load forecast is $25 \%$ more than the maximum. This case is illustrated in Figure 3.

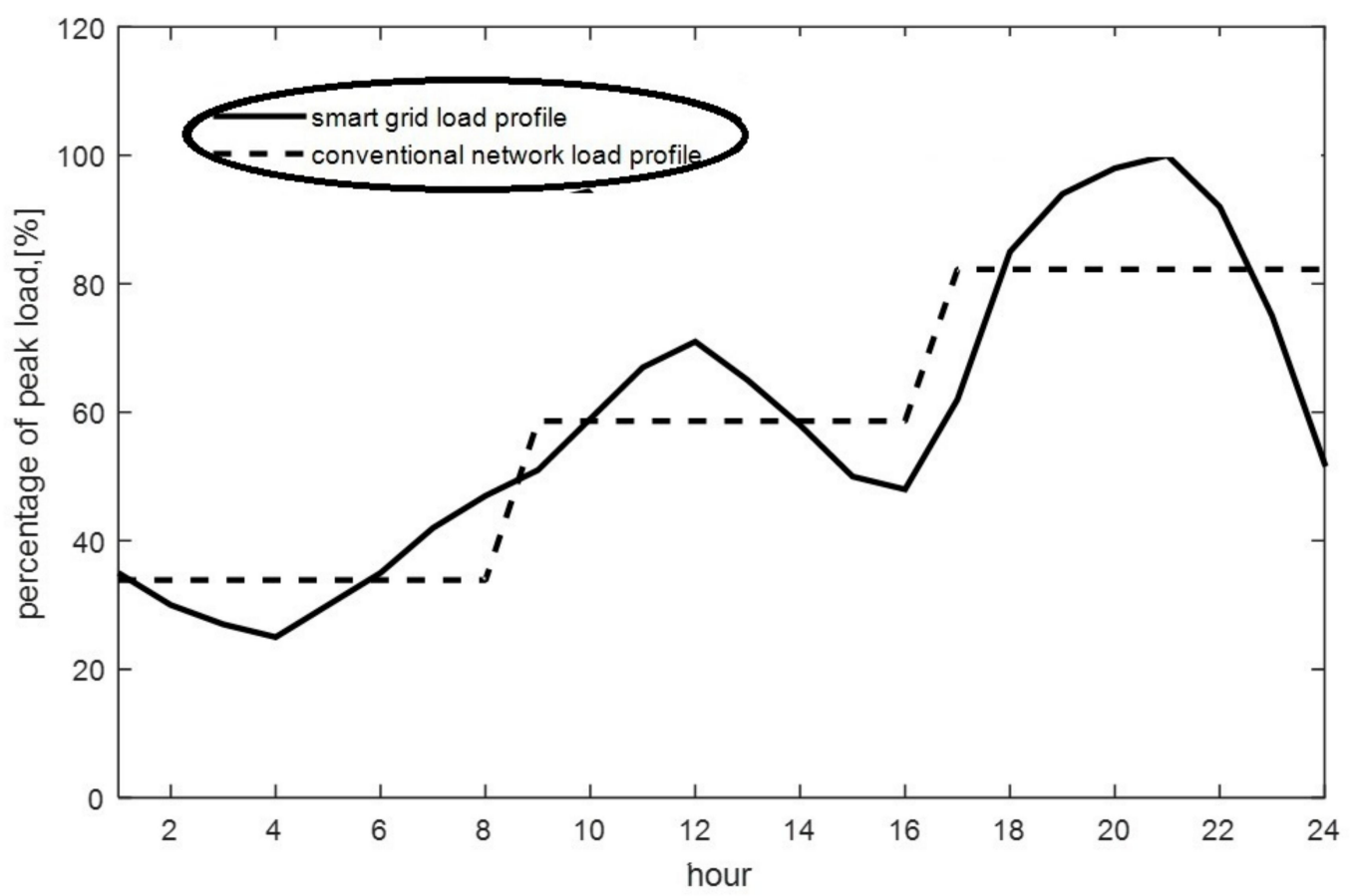

Figure 1. Load model of Cases 1 and 2. 


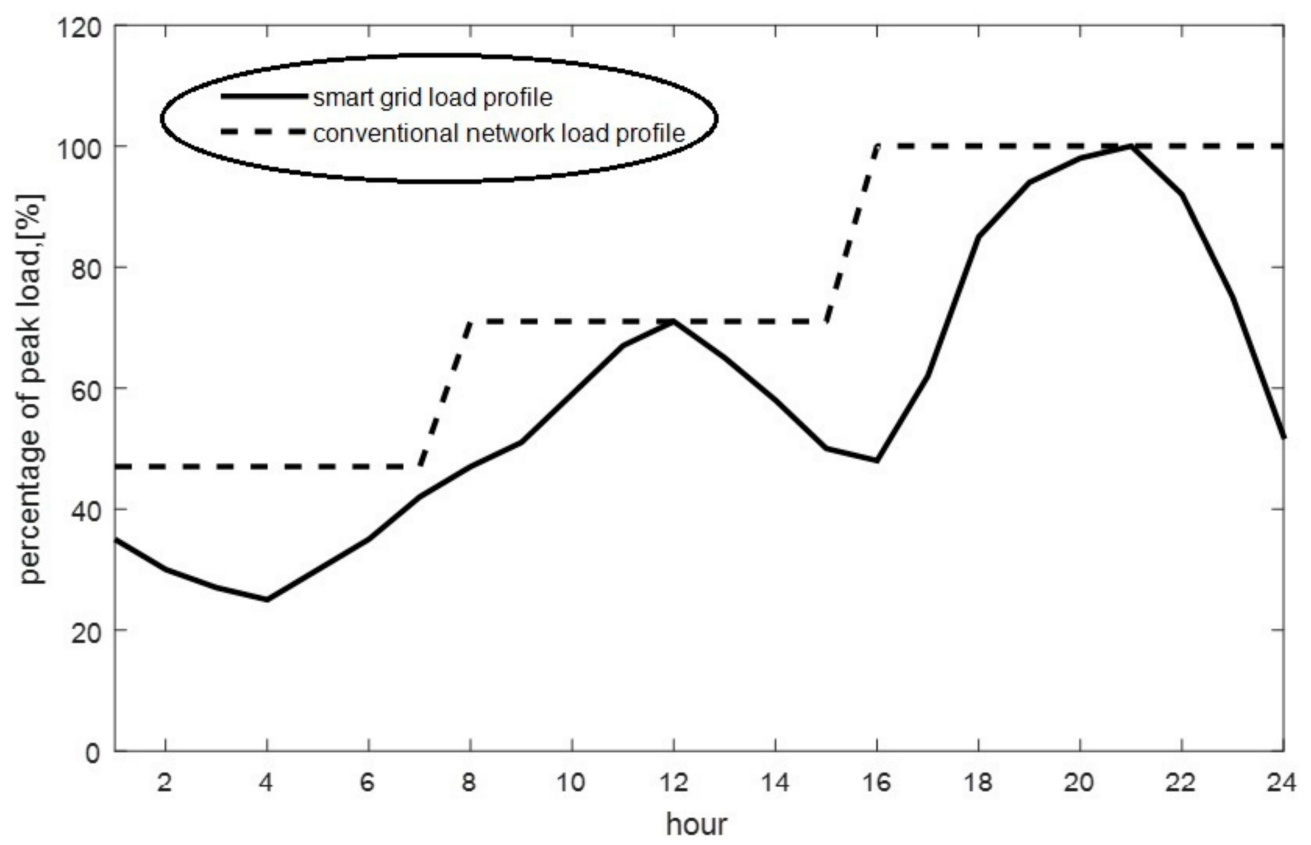

Figure 2. Load model of Cases 1 and 3.

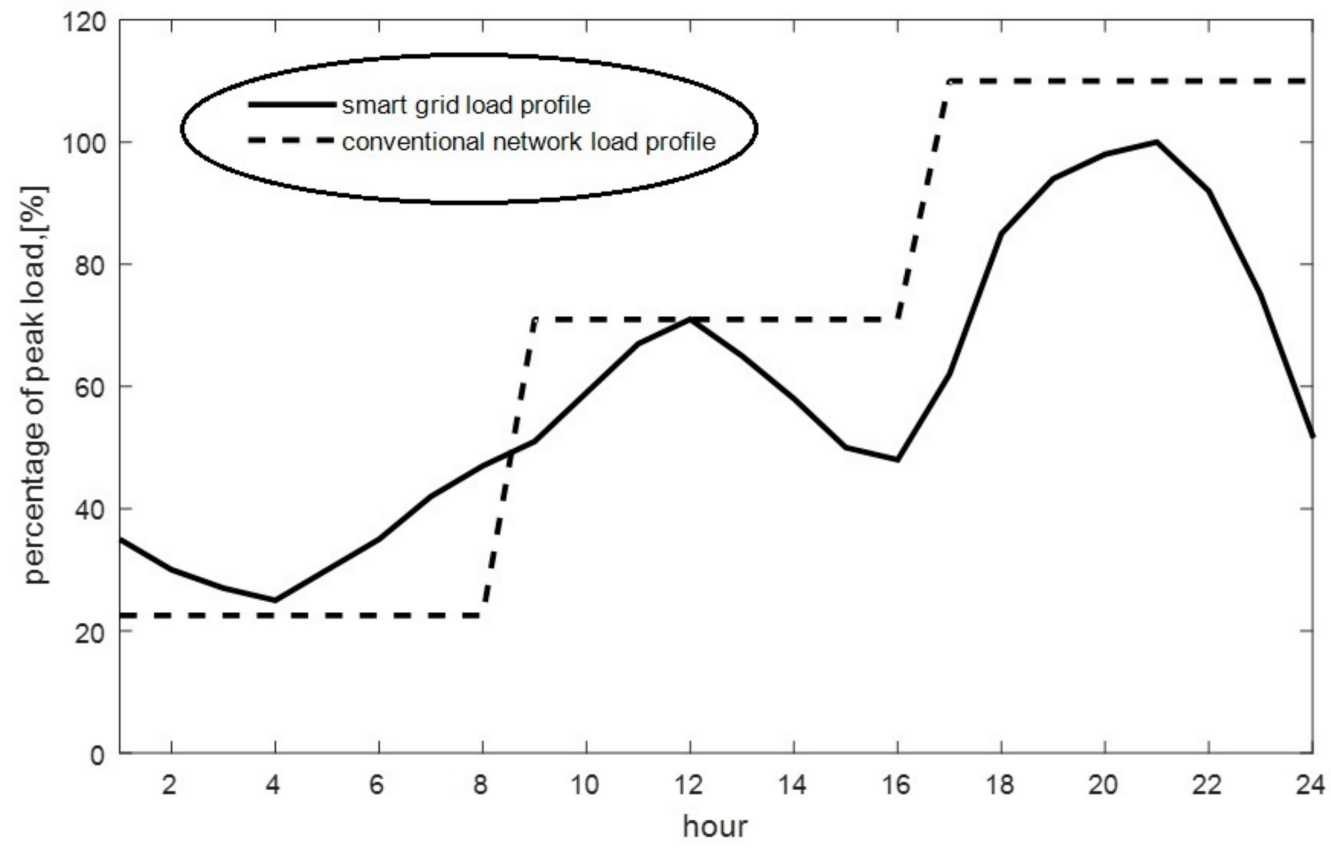

Figure 3. Load model of Cases 1 and 6.

\section{Proposed Optimization Method}

4.1. Introduction of MRFO Method

The MRFO algorithm is inspired by foraging strategies of manta ray, including chained, spinning, and turning. Figure 4 shows the chained, spinning and turning strategies in the MRFO algorithm [31,32]. The mathematical model of each strategy is described. 


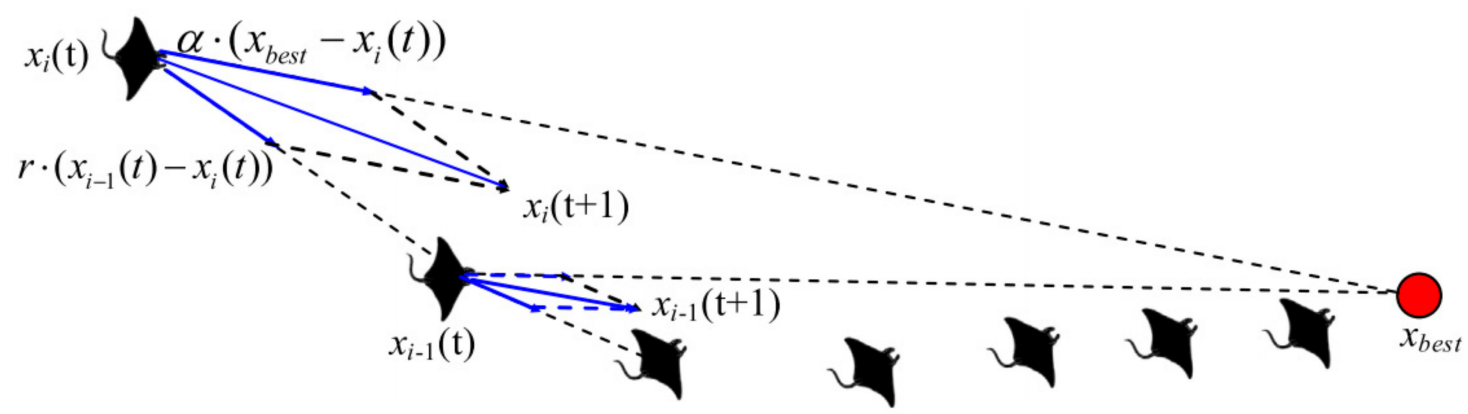

(a)

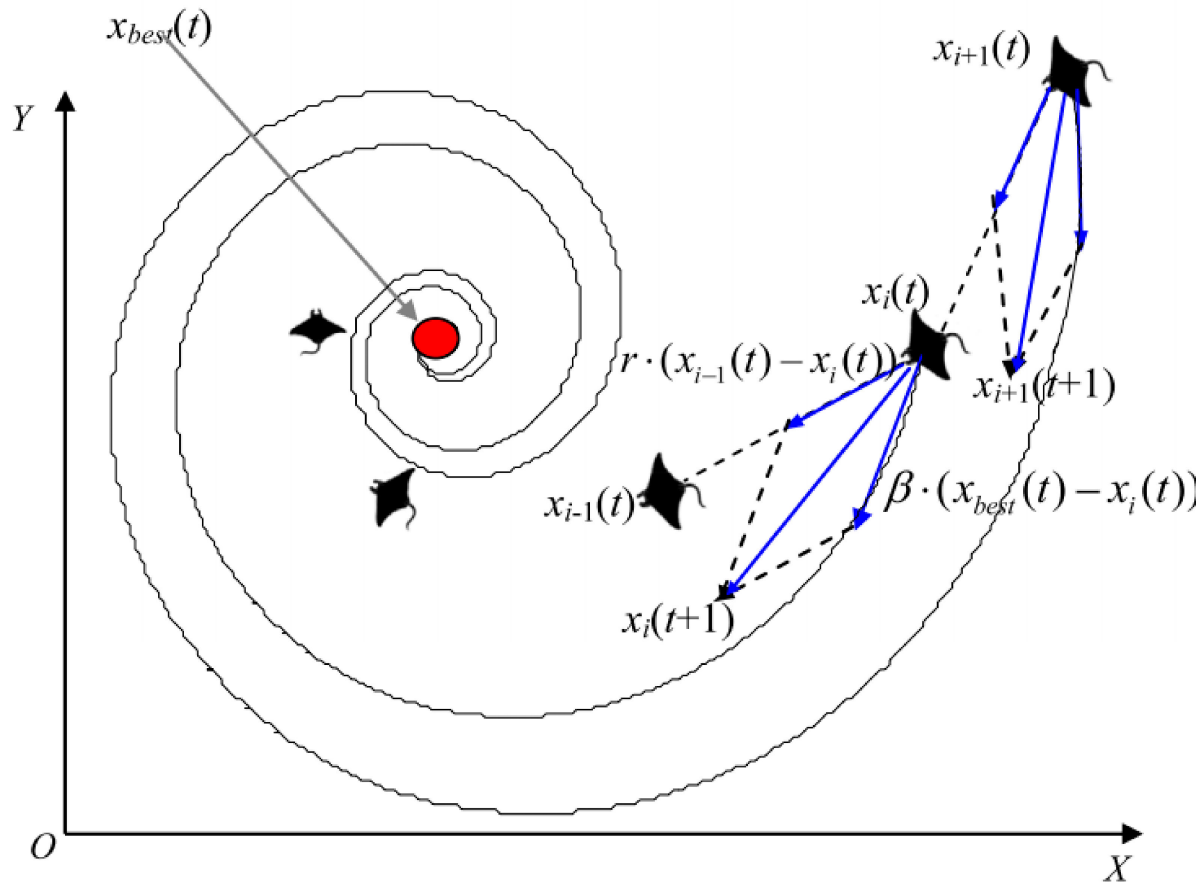

(b)

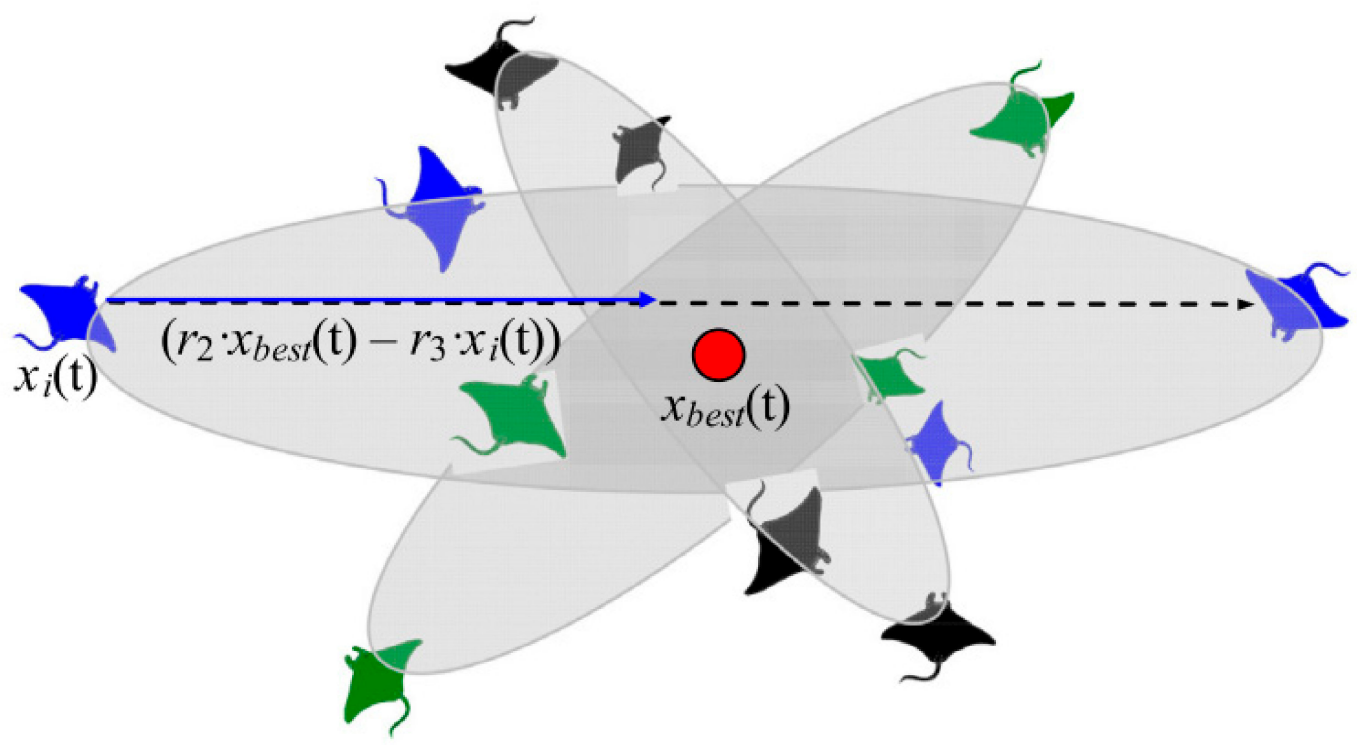

(c)

Figure 4. Strategies of MRFO algorithm (a) chain (b) cyclone and (c) somersault [31]. 


\subsubsection{Foraging with Chain Strategy}

In the MRFO algorithm, manta rays follow plankton by tracking its position. A better position indicates more plankton in that position. In each iteration, each manta ray is updated based on the best current solution and the solution before it is updated. The mathematical model of chained search strategy is defined as follows [31,32]:

$$
\begin{gathered}
x_{i}^{d}(t+1)=\left\{\begin{array}{cl}
x_{i}^{d}(t)+r \cdot\left(x_{\text {best }}^{d}(t)-x_{i}^{d}(t)\right)+\alpha \cdot\left(x_{\text {best }}^{d}(t)-x_{i}^{d}(t)\right) & i=1 \\
x_{i}^{d}(t)+r \cdot\left(x_{i-1}^{d}(t)-x_{i}^{d}(t)\right)+\alpha \cdot\left(x_{\text {best }}^{d}(t)-x_{i}^{d}(t)\right) & i=2, \ldots, N
\end{array}\right. \\
\alpha=2 \cdot r \cdot \sqrt{|\log (r)|}
\end{gathered}
$$

where, $x_{i}^{d}(t)$ indicates $i$ th manta ray position in $d$ th dimension at $\mathrm{t}, \mathrm{r}$ is a vector in the distance $[0,1]$ randomly, a refers to a weight coefficient, and $x_{\text {best }}^{d}(t)$ is the plankton with the largest density.

\subsubsection{Foraging with Cyclone Strategy}

In the cyclone strategy, the manta rays, in addition to the spinning movement towards the food, also move towards one of the manta rays in front of it. That is, the manta ray groups have a spiral motion toward the food. The spinning strategy in an n-dimensional space is mathematically defined as follows [31,32]:

$$
\begin{gathered}
x_{i}^{d}(t+1)=\left\{\begin{array}{cl}
x_{\text {best }}^{d}(t)+r \cdot\left(x_{\text {best }}^{d}(t)-x_{i}^{d}(t)\right)+\beta \cdot\left(x_{\text {best }}^{d}(t)-x_{i}^{d}(t)\right) & i=1 \\
x_{\text {best }}^{d}(t)+r \cdot\left(x_{i-1}^{d}(t)-x_{i}^{d}(t)\right)+\beta \cdot\left(x_{\text {best }}^{d}(t)-x_{i}^{d}(t)\right) & i=2, \ldots, N
\end{array}\right. \\
\beta=2 e^{r_{1} \frac{T-t+1}{T} \cdot \sin \left(2 \pi r_{1}\right)}
\end{gathered}
$$

where, $\beta$ is the weighting factor, $T$ refers to the maximum iteration number and $r_{1}$ is a random number in the distance $[1,0]$.

All manta rays randomly perform a search for food according to their reference position, so the spinning search represents a desirable exploration for the area with best solution. This advantage allows the the MRFO to have access to a wide global search, which is presented as follows [31,32]:

$$
\begin{aligned}
& x_{\text {rand }}^{d}=L b^{d}+r \cdot\left(U b^{d}-L b^{d}\right) \\
& x_{i}^{d}(t+1)= \begin{cases}x_{\text {rand }}^{d}+r \cdot\left(x_{\text {rand }}^{d}-x_{i}^{d}(t)\right)+\beta \cdot\left(x_{\text {rand }}^{d}-x_{i}^{d}(t)\right) & i=1 \\
x_{\text {rand }}^{d}(t)+r \cdot\left(x_{i-1}^{d}(t)-x_{i}^{d}(t)\right)+\beta \cdot\left(x_{\text {rand }}^{d}-x_{i}^{d}(t)\right) & i=2, \ldots, N\end{cases}
\end{aligned}
$$

where, $x_{\text {rand }}^{d}$ is random position, $L b^{d}$ and $U b^{d}$ are the minimum and maximum bounds for dimension $d$, respectively.

\subsubsection{Foraging with Somersault Strategy}

In this strategy, the food position is a pivot. Each manta ray tries swimming around the axis and back to a new position, so the manta rays always maintain their position around the current best position. This strategy is defined by $[31,32]$ :

$$
x_{i}^{d}(t+1)=x_{i}^{d}(t)+S \cdot\left(r_{2} \cdot x_{\text {best }}^{d}-r_{3} \cdot x_{i}^{d}(t)\right), i=1, \ldots, N
$$

where, $S$ is the somersault factor (equal to 2), $r_{2}, r_{3}$ represent random numbers in the range $[0,1]$.

In each iteration, each manta ray updates its position relative to the two manta rays in front of it, as well as to the reference position. To perform the exploration and exploitation search, the $t / T$ value reduces from $1 / T$ to 1 . In the case of $t / T<$ rand, the best current position is selected as the reference position in exploitation phase, for $\mathrm{t} / \mathrm{T}>$ rand a position is considered randomly as the reference position in the search space for the exploration 
phase. The MRFO method has the ability to switch between chain and cyclone strategies. The position of the manta ray can also be updated to the current best position based on the somersault strategy. The proposed steps and the update step are continued to establish the convergence conditions of the MRFO method, and finally the best position and the corresponding amount of fitness are determined. Flowchart of the MRFO algorithm is presented in Figure 5.

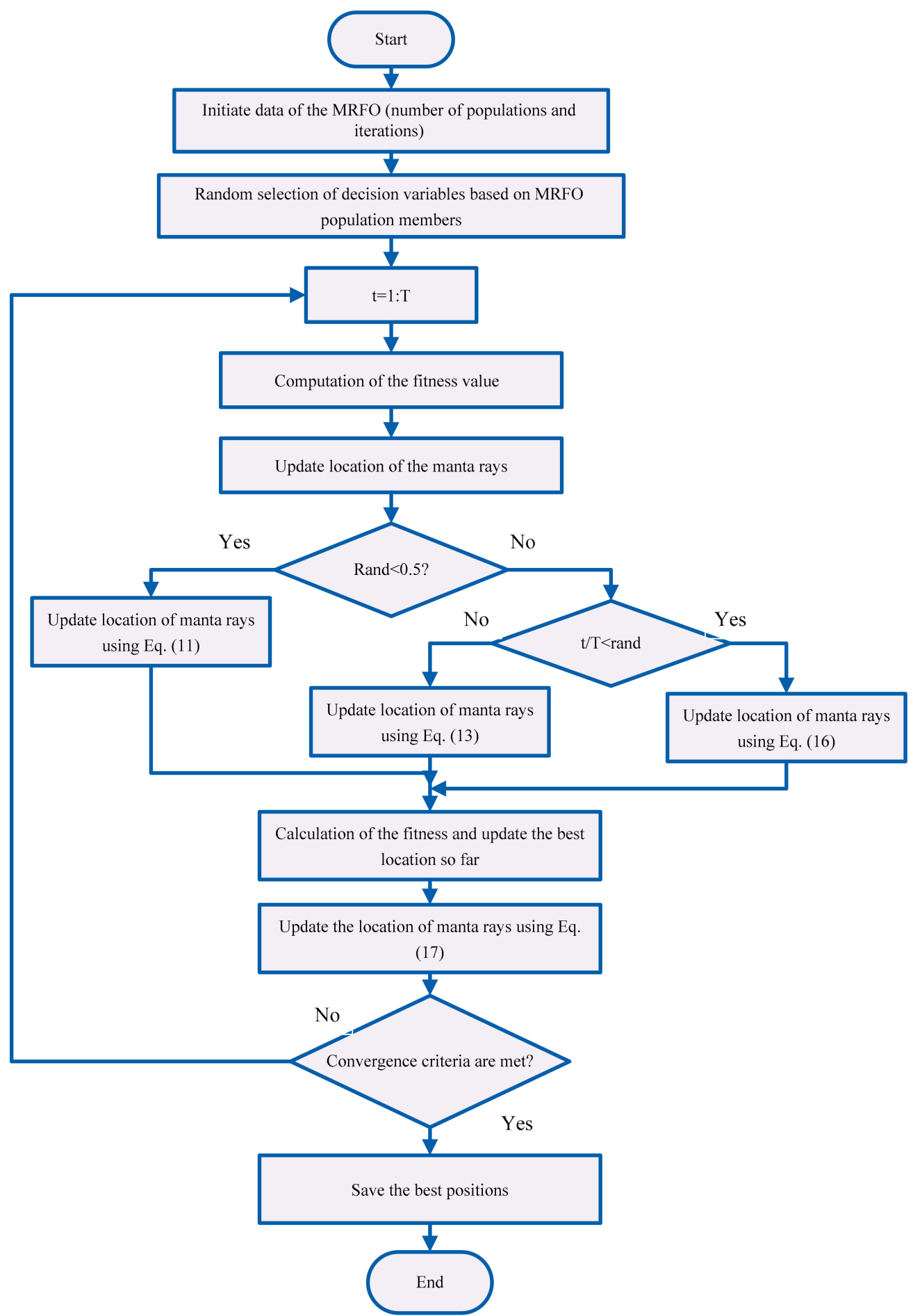

Figure 5. Flowchart of the MRFO algorithm [31]. 


\subsection{MRFO Based Planning}

After the optimal allocation of DGSCs in the network and the optimal distribution of their power, using the backward-forward load flow, the current of lines and the voltage deviations of the network buses are obtained. Therefore, the program easily calculates the amount of reduction of network losses and also the reduction of voltage deviations of the buses and finally, effect of improving the optimal application of the DGSCs in the total objective function value can be determined. Moreover, in the problem-solving procedure, the program constantly checks the network operation constraints so that the voltage and current are within the allowable range. It should be noted that when executing the planning problem in case of violation of the constraints, the program keeps the constraints within its permissible range by changing the size of the DGSCs in the planning problem. So, the optimization program in solving the planning problem determines the optimal distribution of the DGSCs power in such a way that the energy losses of the study period is minimized and of course the operating constraints are also met.

The flowchart of the implementation of the MRFO in allocation of the DGSCS in the network is depicted in Figure 6. After allocation of the DGSCs in the network, after finding the installation location of the DGSCs using the planning program the optimal distribution of the DGSCs power is found. The steps for using DGSCs based on the MRFO in planning problem are as follows:

(Step 1) The data of the distribution network including loads and lines data, location and maximum size of DGSCs (obtained from the DGSCs allocation problem) as well as the load model are applied to the program based on the MRFO method. The parameters of the MRFO are also set. The population, maximum iteration and also repetition are considered 50, 100 and 30, respectively based on trial-and-error method.

(Step 2) Based on the provided load cases, a load level is evaluated.

(Step 3) In this step, the optimal planning of DGSCs is implemented. After evaluating the operating constraints, the objective function as energy losses (Equation (5)) is calculated based on the optimal operating point according to the load level considering the constraints (Equations (6)-(8)) in step 2.

(Step 4) All the load levels are checked. In this case, go to next step, otherwise step 2 must be performed.

(Step 5) The objective function as energy losses (Equation (5)) is determined for 24-h for the network.

(Step 6) The optimal distribution of the DGSCs power is determined and stop the algorithm. 


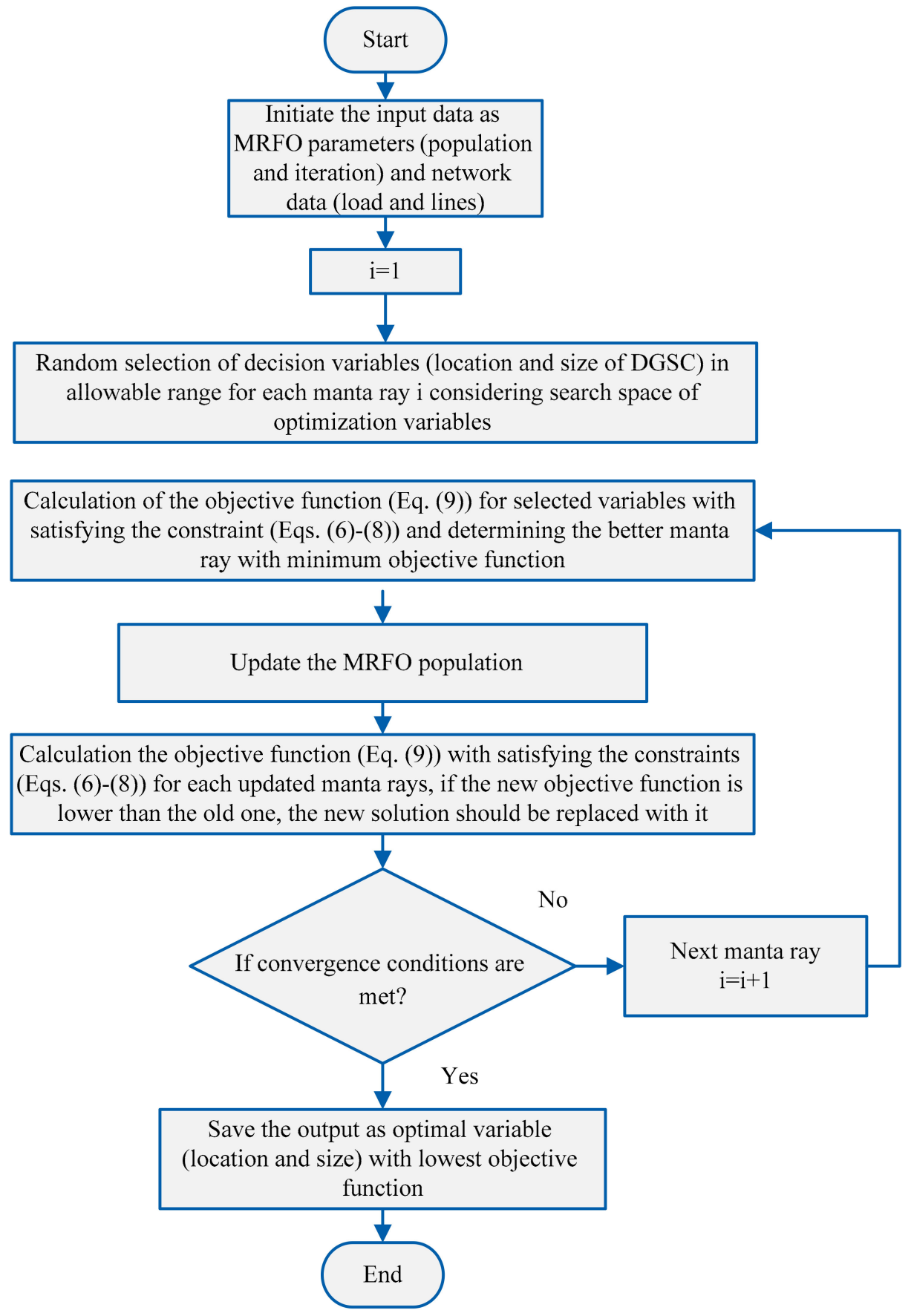

Figure 6. Implementation of the MRFO in allocation of the DGSCS in the network.

\section{Simulation Results}

\subsection{Network Data}

In this study, the optimal allocation and planning of power generation resources the DGSCs is presented in smart environment with objective of reducing losses and considering the voltage deviations minimization via the MRFO. Figure 7 shows the IEEE 69-bus network. Load data and network lines are derived from [34]. The network power loss in peak load conditions is equal to $224.97 \mathrm{~kW}$. In this research, MATLAB (R2015a) is applied to implement the methodology based on a personal computer with a Core i7, 3.1 GHz CPU, 8 GB memory, 1 T HDD and the Windows 7 operating system. 


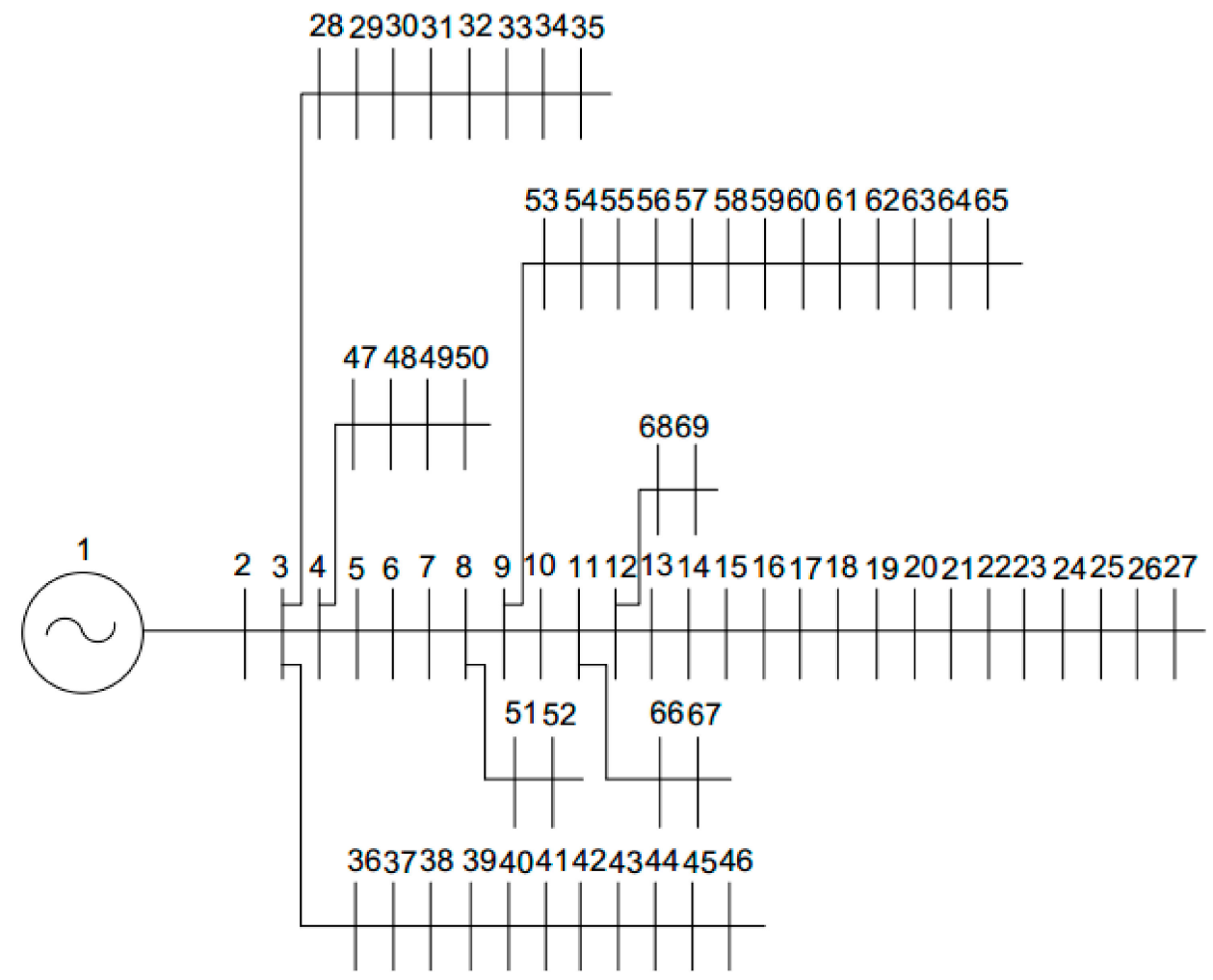

Figure 7. Schematic of IEEE 69-bus distribution network.

\subsection{Optimal Allocation of the DGSCs}

Allocation of the DGSCs is implemented based on peak load conditions via the MRFO. In this study optimal allocation of one and two DGSCs are considered. The allocation problem is performed using the MRFO algorithm and its capability is investigated with PSO. In this study, the PSO with variant inertia weight is used with cognitive component $\mathrm{c}_{1}=1.5$, social component $c_{2}=1.5$, minimum inertia weight $\mathrm{w}_{\min }=0.4$ and maximum inertia weight $\mathrm{w}_{\min }=0.9$. The optimization results are presented first as a single objective with objective of losses minimization and then as a multi-objective method with objective of losses and voltage deviations minimization. The convergence curves of both methods are presented in Figure 8, which confirms the superiority of the MRFO with lower convergence tolerance and lower objective function. The results of single-objective allocation are presented in Table 1. As can be seen, in the case of using one DGSC, the performance of the MRFO and PSO methods is the same in terms of objective function value or the amount of power losses. In single-objective optimization with a DGSC, both methods have chosen bus 50 DGSC installation and for two DGSCs allocation, the proposed methods have also selected buses 50 and 51 for installation of two DGSCs. 


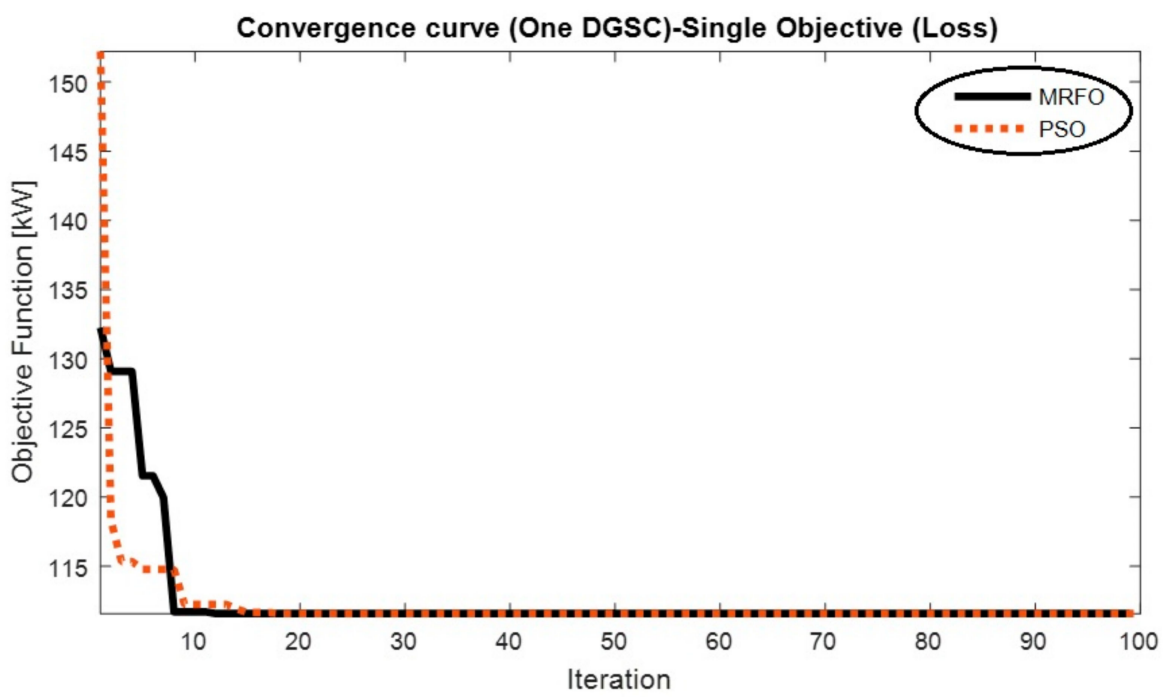

(a)

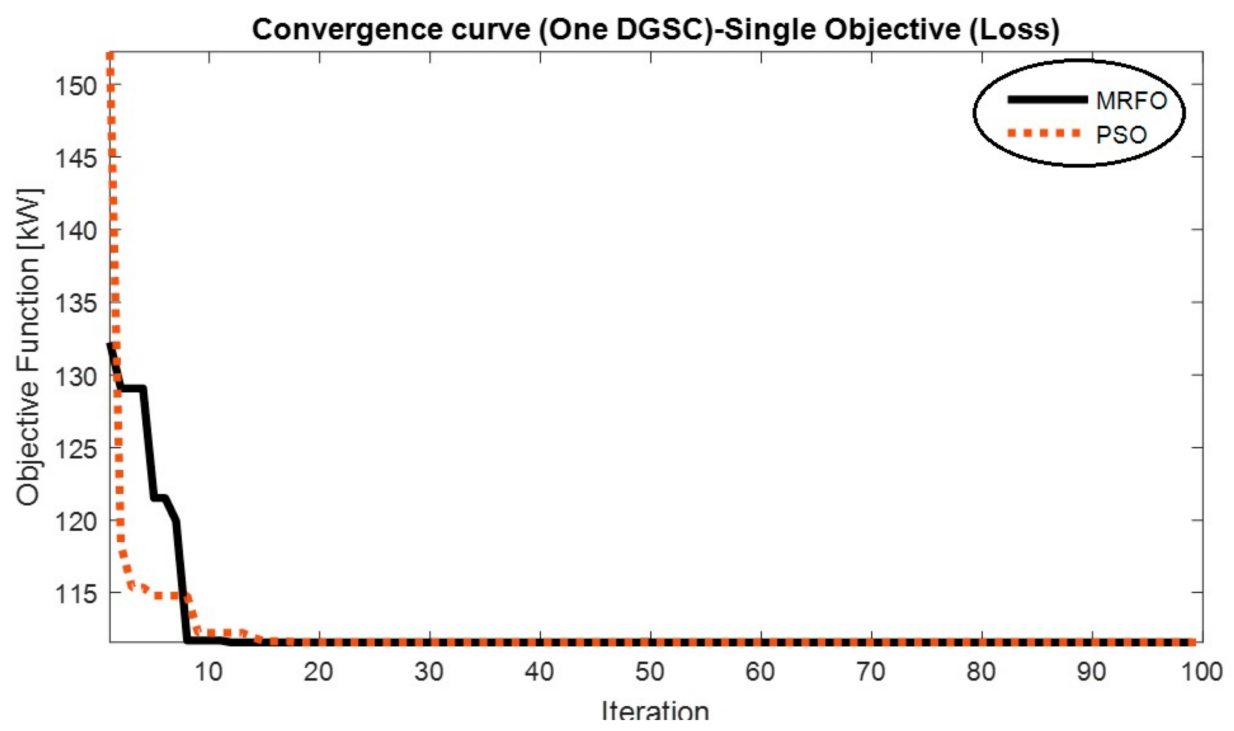

(b)

Figure 8. Convergence curve of MFRO and PSO methods for single-objective allocation of (a) one DGSC (b) two DGSCs.

Table 1. Optimal allocation of DGSCs in IEEE 69-bus network as single-objective optimization.

\begin{tabular}{cccc}
\hline One DGSC & Without DGSC & With DGSC (PSO) & With DGSC (MRFO) \\
\hline Item/Value & & PSO & MRFO \\
\hline Location (Bus) & - & 50 & 50 \\
Size (kW) & - & 998.96 & 1000 \\
Power Loss (kW) & 202.67 & 111.59 & 111.58 \\
Minimum Voltage (p.u) & 0.9038 & 0.948 & 0.9482 \\
\hline Two DGSC & Without DGSC & With DGSC (PSO) & With DGSC (MRFO) \\
\hline Item/Value & - & PSO & MRFO \\
\hline Location (Buses) & - & 50,51 & 50,51 \\
Sizes (kW) & 202.67 & 1000,860 & 1000,865 \\
Power Loss (kW) & 0.9038 & 83.370 & 83.330 \\
\hline Minimum Voltage (p.u) & 0.9681 & 0.9683 \\
\hline
\end{tabular}


It should be noted that the results obtained from the allocation of the DGSCs including selected buses in single-objective optimization based on the MRFO method are similar to the PSO results. Moreover, the losses value for one DGSC allocation is obtained $111.58 \mathrm{~kW}$ and $11.59 \mathrm{~kW}$, also the minimum voltage is achieved $0.9482 \mathrm{p} . \mathrm{u}$ and $0.9480 \mathrm{p} . \mathrm{u}$, respectively using the MRFO and PSO methods. Also, the losses value for two DGSCs allocation is achieved $83.34 \mathrm{~kW}$ and $83.37 \mathrm{~kW}$, and the minimum voltage is obtained 0.9683 p.u and 0.9681 p.u, respectively using the MRFO and PSO methods, so the results prove the better performance of the MRFO in achieving to lower losses and better voltage profile with lower convergence tolerance and more convergence rate than the PSO.

Also, the voltage profile for one and two DGSCs is shown in Figure 9, which shows that increasing the number of DGSCs improves the voltage profile or reduces the in the network.

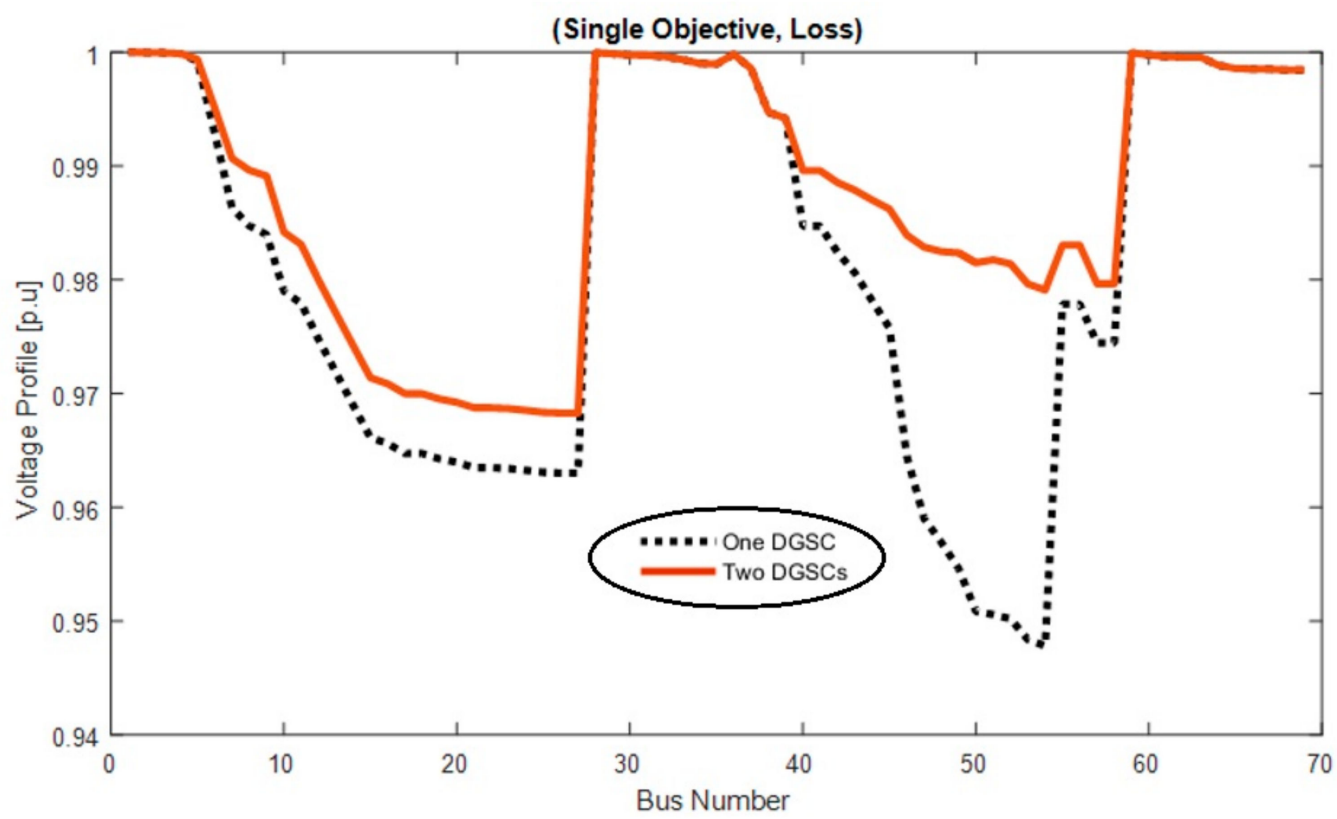

Figure 9. Voltage profile of the 69-bus network for one and two DGSCs using MRFO.

In Figure 10, the convergence process of problem solving is presented as multiobjective allocation for one and two DGSCs.

According to Figure 10, in multi-objective allocation, the MRFO method obtained the same power loss value for one DGSC allocation with the PSO and also lower losses value for two DGSCs allocation than the PSO. Statistical analysis of the MRFO and PSO performance in multi-objective allocation problem solving in view of the best, mean and worst values of the objective function based weighted coefficients method is presented in Table 2. As we know, the value of the objective function is normalized and is in the range of 0 to 1 . The results of Table 2, showed the better performance of the MRFO in achieving to lowest value of the objective function in 30 repetitions of the optimization algorithms with better results of the best, mean, worst and standard deviation (St. D.) values in allocation of one and two DGSCs allocation than the PSO. 


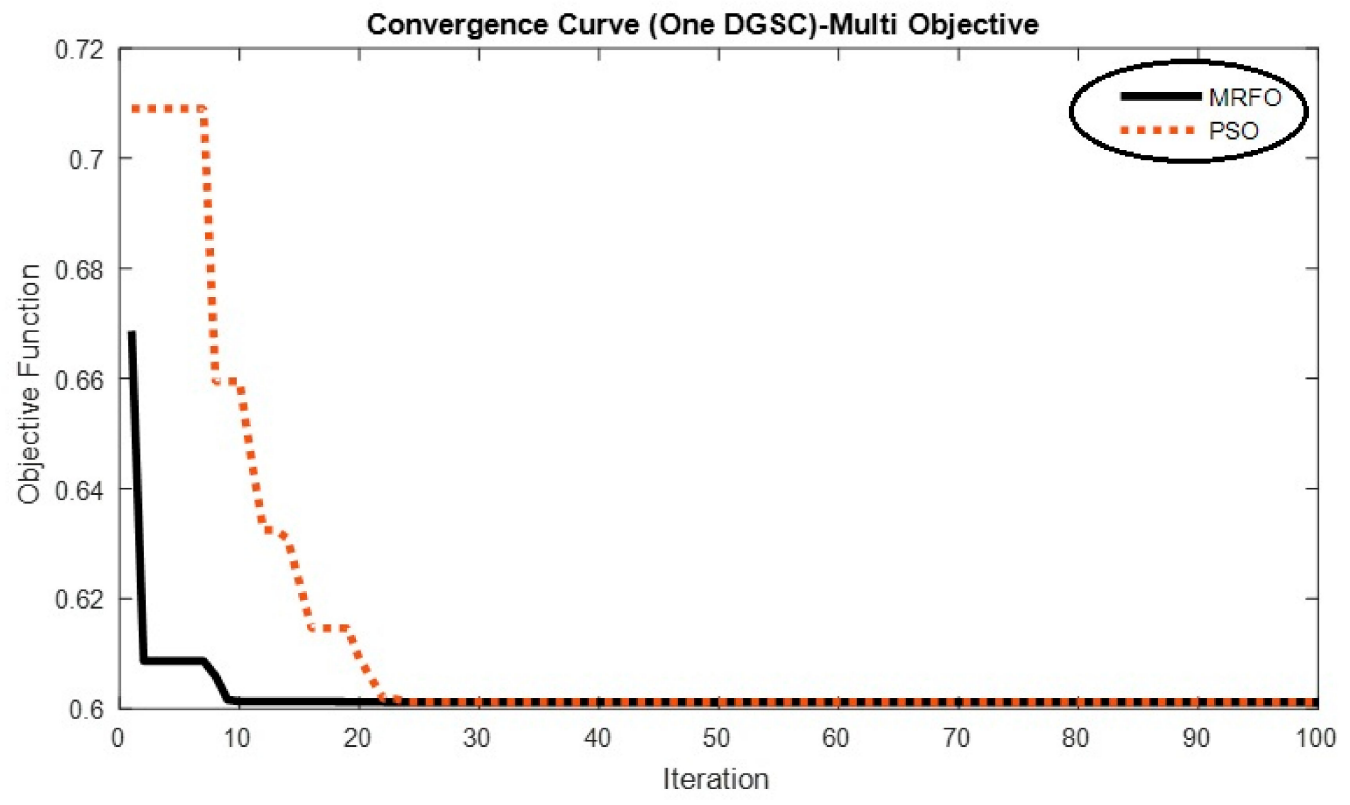

(a)

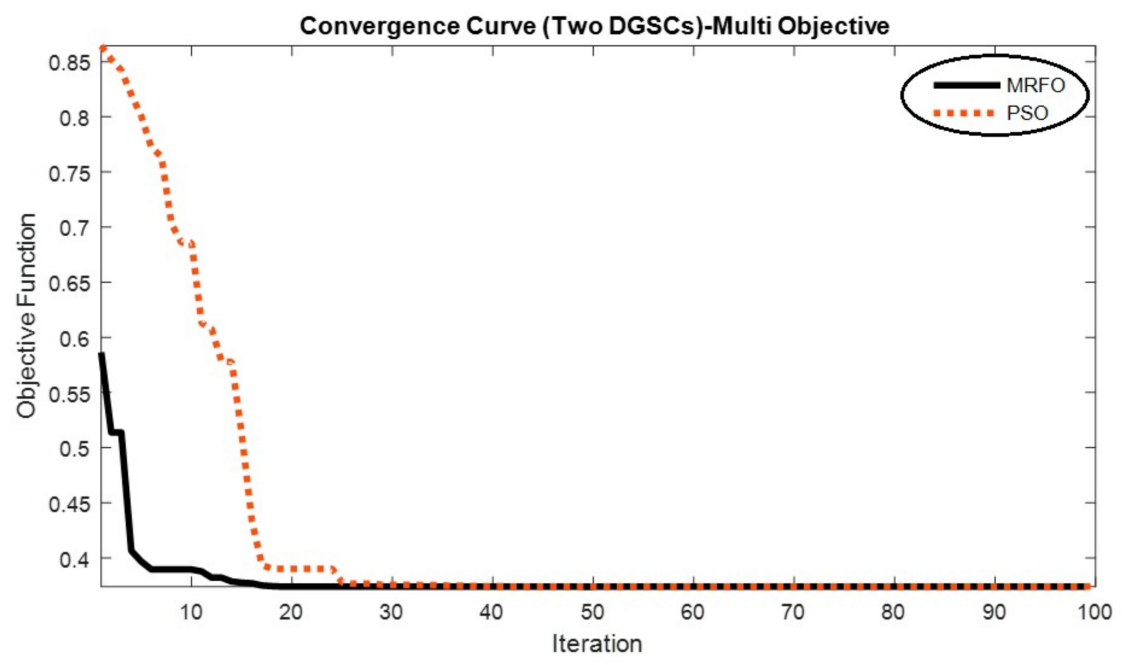

(b)

Figure 10. Convergence curve of MFRO and PSO methods for multi-objective allocation of (a) one DGSC (b) two DGSCs.

Table 2. Statistical analysis of the MRFO and PSO performance in allocation problem solving.

\begin{tabular}{cccccc}
\hline Allocation Problem & Method & Best (MUS\$) & Mean (MUS\$) & Worst (MUS\$) & St. D. (\$) \\
\hline \multirow{2}{*}{ One DGSC } & MRFO & 0.6015 & 0.6033 & 0.6076 & 0.247 \\
\multirow{2}{*}{ Two DGSCs } & PSO & 0.6027 & 0.6048 & 0.6090 & 0.261 \\
& MRFO & 0.3511 & 0.3519 & 0.3527 & 0.118 \\
\hline
\end{tabular}

The multi-objective optimization results are given in Table 3 . In the allocation of one DGSC, both methods chose bus 54 for installation, while in single-objective optimization, both methods determined bus 50 for DGSC installation. In the optimal allocation of two DGSCs, the MRFO selected buses 50 and 52 and the PSO method selected buses 50 and 12 to install the DGSCs. The voltage profile for multi-objective allocation of one and two DGSCs is shown in Figure 11. The voltage profiles for single and multi-objective location 
of one and two DGSC are also shown in Figures 11 and 12, respectively. Here, according to Figures 11-13, it can be concluded that increasing the number of DGSCs improves the voltage profile. Also, in multi-objective allocation, the network voltage is more improved that the single-objective allocation, which makes it important to use multiple indices.

Table 3. Results of multi-objective allocation and sizing of one and two DGSCs in a 69-bus network.

\begin{tabular}{cccc}
\hline One DGSC & Without DGSC & With DGSC (PSO) & With DGSC (MRFO) \\
\hline Item/Value & & PSO & MRFO \\
\hline Location (Bus) & - & 54 & 54 \\
Size (kW) & - & 1000 & 1000 \\
Power Loss (kW) & 202.67 & 121.539 & 121.539 \\
Minimum Voltage (p.u) & 0.9038 & 0.9500 & 0.9500 \\
\hline Two DGSC & Withoud DGSC & With DGSC (PSO) & With DGSC (MRFO) \\
\hline Item/Value & - & PSO & MRFO \\
\hline Location (Buses) & - & 50,12 & 50,52 \\
Sizes (kW) & 202.67 & 1000,1000 & 1000,965 \\
Power Loss (kW) & 0.9038 & 85.493 & 83.603 \\
Minimum Voltage (p.u) & 0.9691 & 0.9694 \\
\hline
\end{tabular}

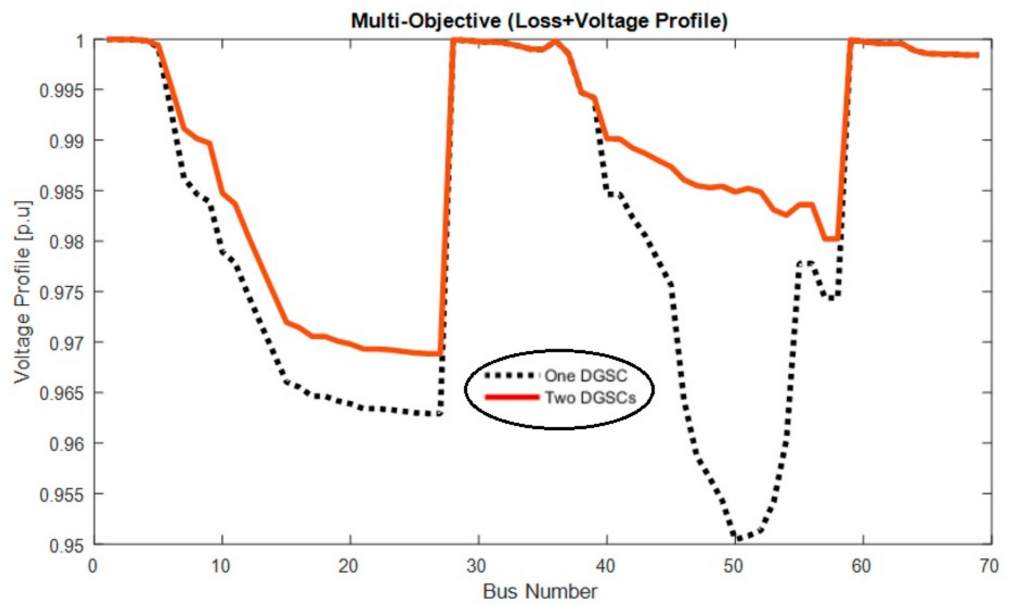

Figure 11. Voltage profile of 69-bus network for multi-objective optimization of one and two DGSCs using MRFO.

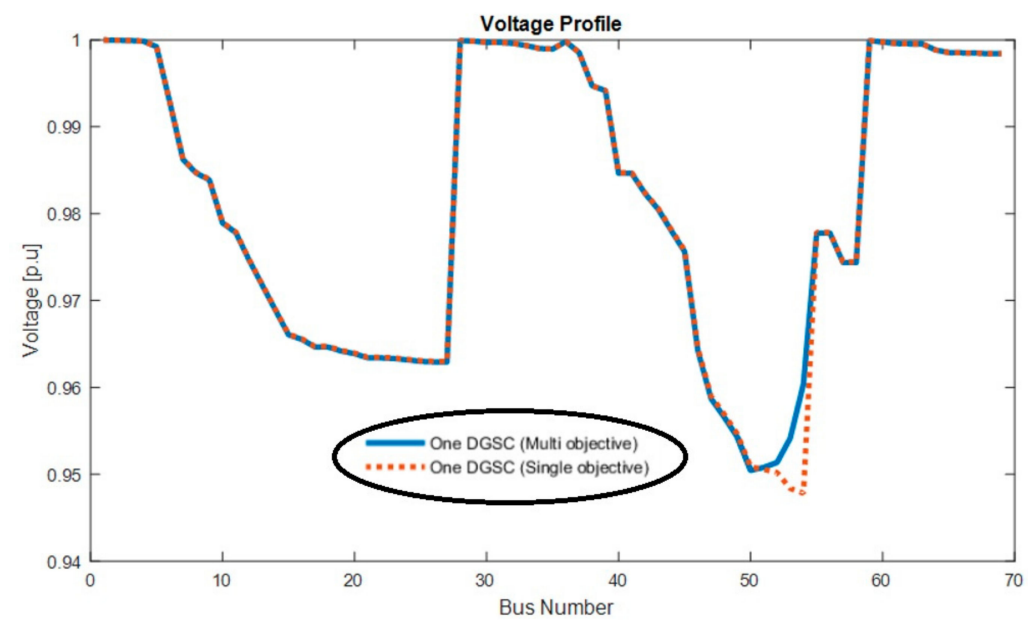

Figure 12. Voltage profile of 69-bus network for single and multi-objective optimization of one DGSC using MRFO. 


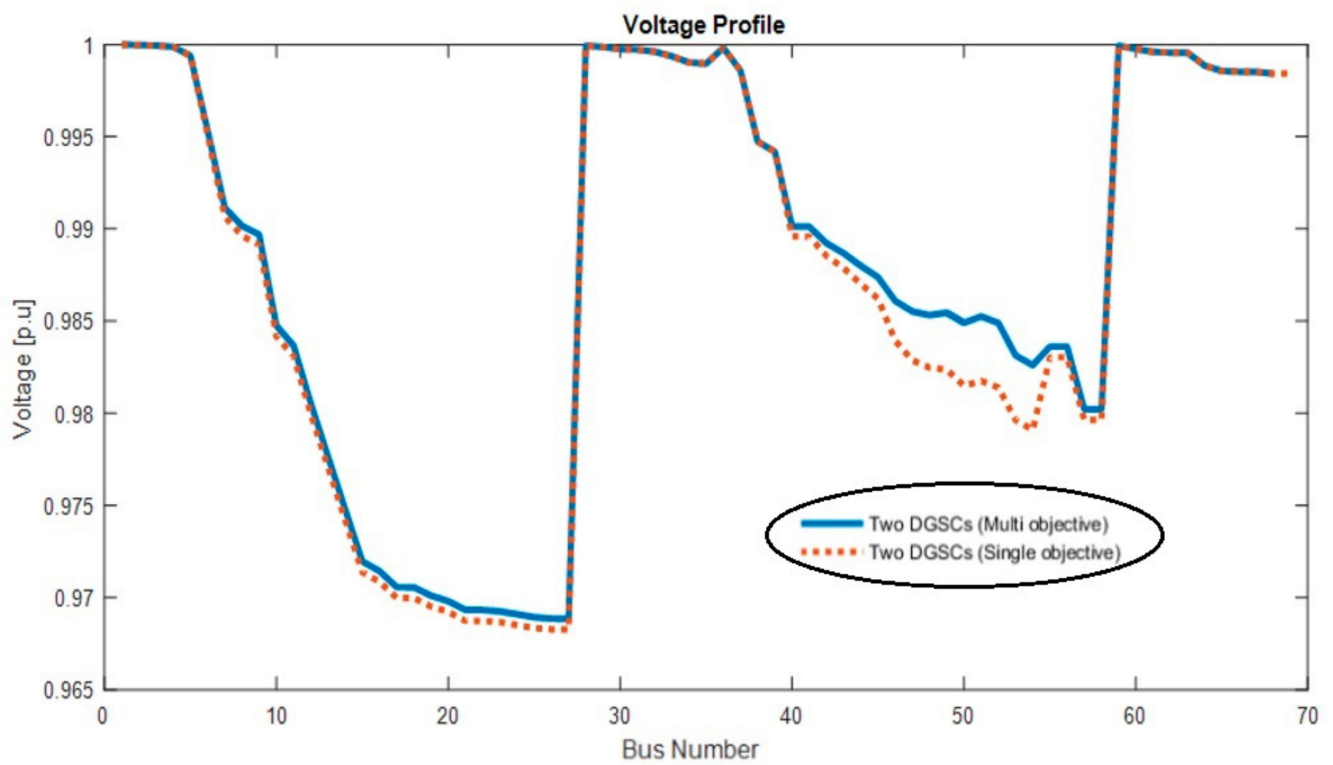

Figure 13. The voltage profile of the network considering two DGSCs via MRFO.

\subsection{Optimal DGSC Planning}

Planning One DGSC (Case 1)

The optimal planning of one DGSC using the MRFO is presented in the first load case considering smart load in Figure 14. The base results of the 69-bus network demand show that loss of energy for the 24-h is obtained $2023 \mathrm{kWh}$. By installing one DGSC in bus 54 (obtained from the allocation problem) and by implementing the optimal planning of DGSC generation in the smart network, the total energy loss is reduced to $1259.39 \mathrm{~kW}$ (37.74\% reduction). A comparison of system hourly energy losses before and after DGSC installation is depicted in Figure 15. Given this figure, it can be argued that the reduction of energy losses is significant, especially during peak times.

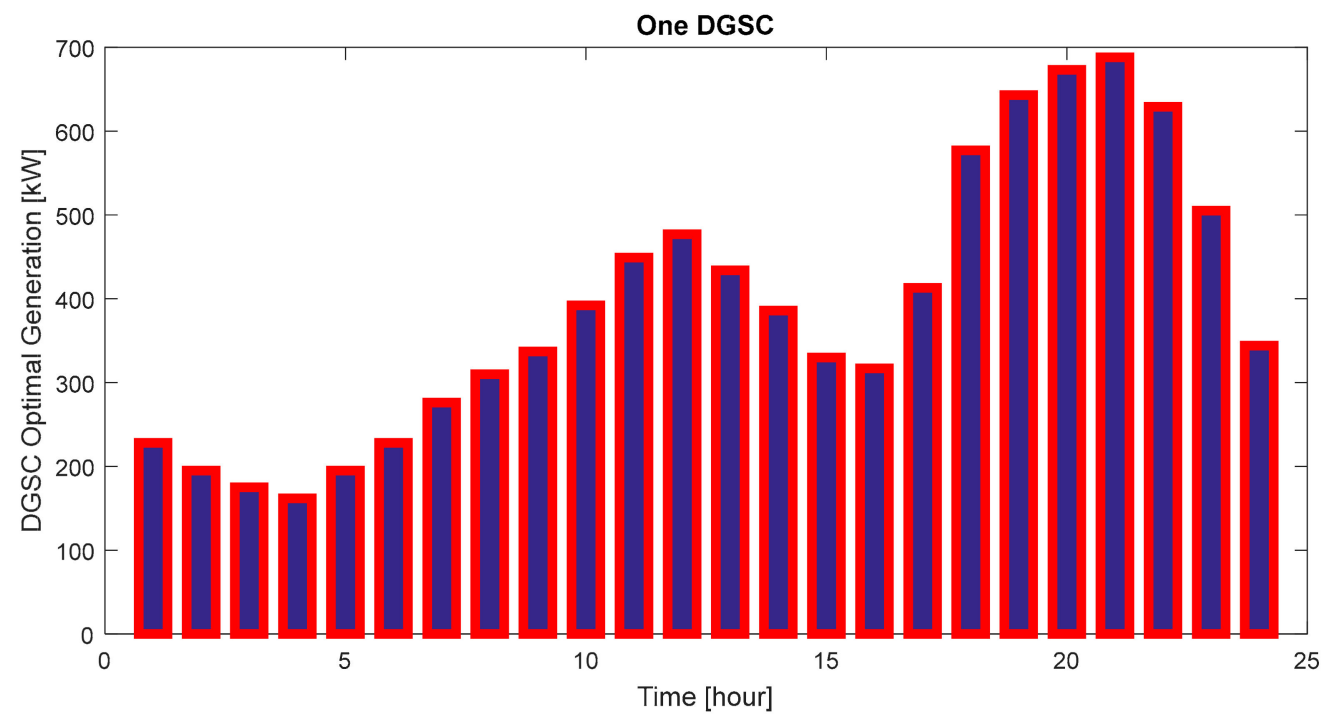

Figure 14. Optimal generation of one DGSC in the case 1. 


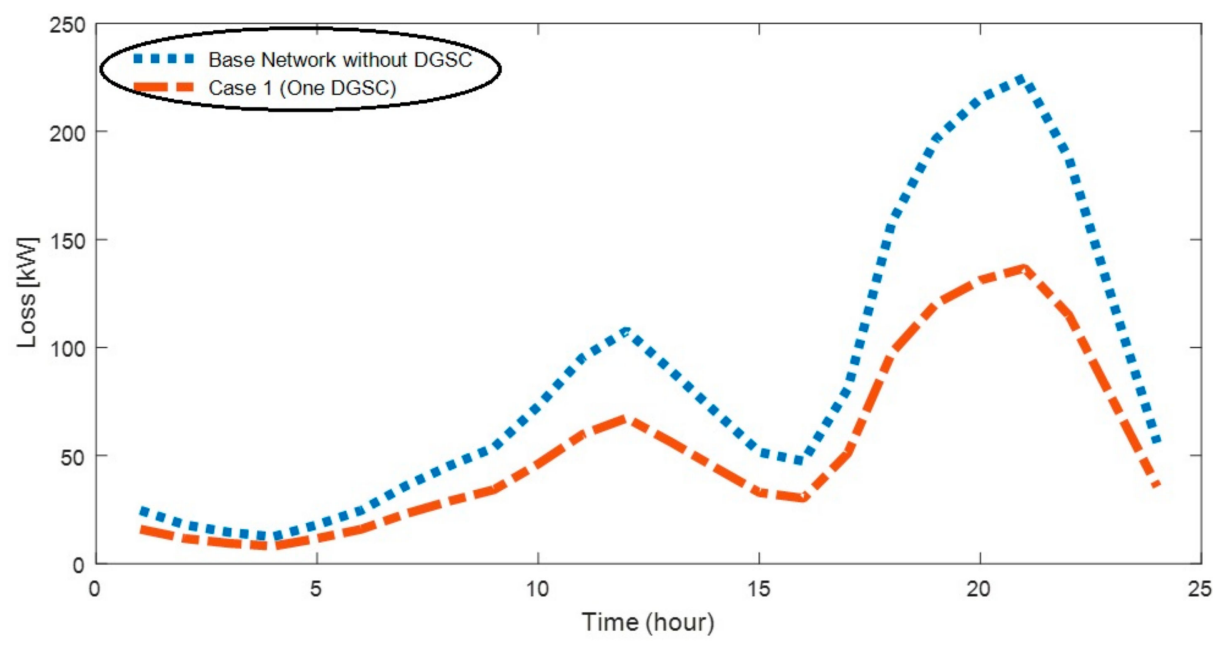

Figure 15. Comparison between network hourly energy losses before and after optimal planning of one DGSC in case 1.

In Case 2, the loss of energy during a day is equal to $1267.52 \mathrm{kWh}$ and this value is $0.64 \%$ more than the value obtained in the smart network. The achieved results are clear in that with the correct data on the demand in the smart network, compared to the normal grid with more accurate load forecasting, the reduction in daily losses is $0.63 \%$ more. With such a large estimate, there is not much difference between the network performance in smart environments and conventional networks. In Case 3, using the MRFO, total daily energy losses are calculated $1645.61 \mathrm{kWh}$ and this value is $382 \mathrm{kWh}$ more than the network with smart demand, so considering this case leads to $30.65 \%$ more loss of energy. In Case 4 , the effect of demand estimation is incorrectly estimated. In this case, the demand is $10 \%$ more compared to Case 3. The optimal DGSC distribution based on this case is presented using MRFO. The loss of energy is computed $2032.79 \mathrm{kWh}$. The loss of energy is approximately $61.39 \%$ more compared to this value in smart network, so the demand forecasting error led to an increase of the loss of energy in this case. In Case 5, the effect of incorrect estimation of the demand as 10\% less than the demand in Case 3, as evaluated in the loss of energy during a day. The results of one DGSC scheduling using the MRFO method show that the daily energy losses are reduced to $1285.40 \mathrm{kWh}$ and this value is $2 \%$ more than the loss of energy in the smart network.

In Case 6, the forecasted demand is defined as multi-level. The energy losses of this case with optimal management of one DGSC using MRFO method is equal to $1759.10 \mathrm{MW}$, which is $39.68 \%$ more than the loss of energy during a day in the smart network case. Therefore, having more accurate demand information has improved network performance. The output difference of DGSC generation in different cases compared with generation with smart demand is shown in Figure 16. The worst case is related to cases 4 and 6 when the demand forecasting error is higher than the smart demand. The higher difference is related to Case 4 and the lowest value is obtained in Case 2. A 10\% load estimation error leads to a $100 \%$ difference from the optimal operational point. The greater difference from the optimum point, the less reduction in losses occurs. Therefore, any deviation from the actual load leads to the occurrence of non-optimal operational point and more energy losses. The energy losses and its deviation percentage in different cases with one DGSC is given in Table 4. 


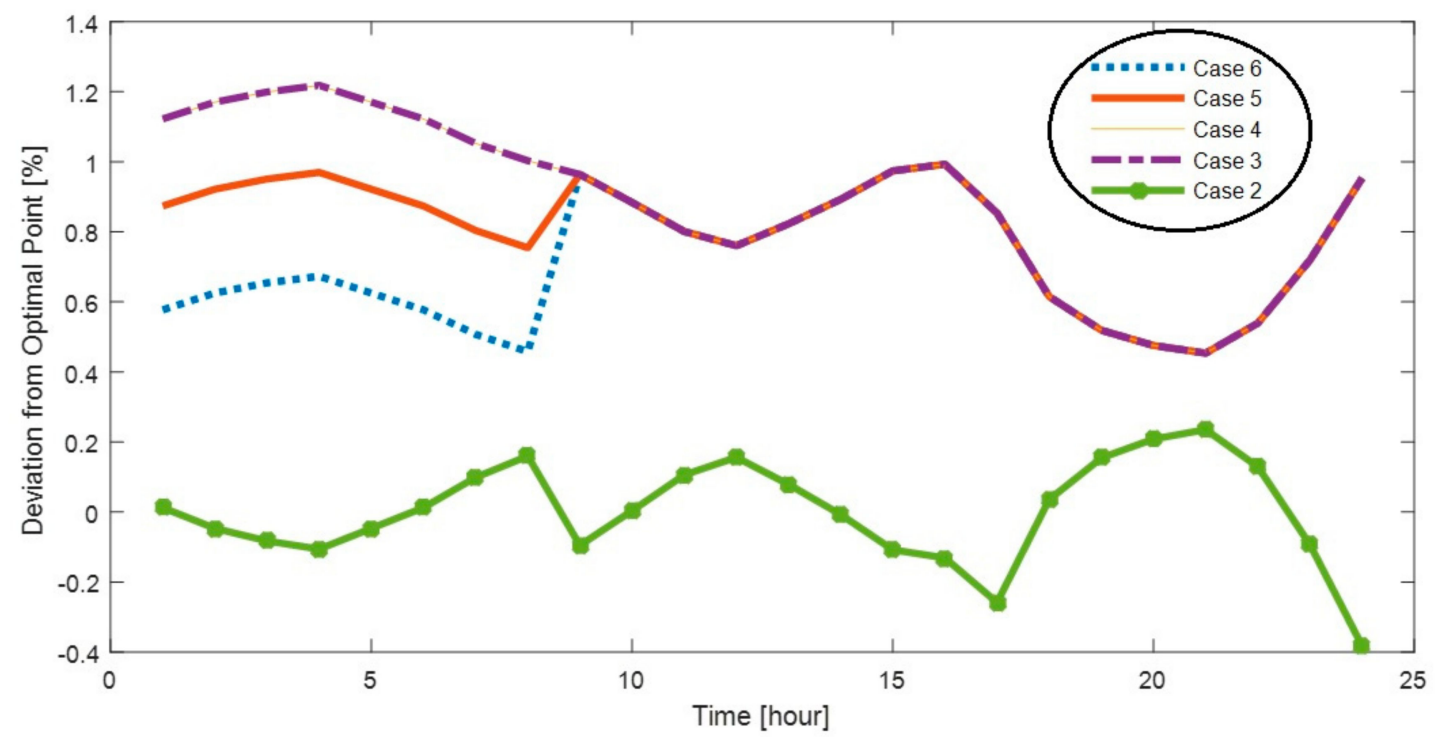

Figure 16. The difference of DGSC generation in conventional network cases from the smart network as optimal point.

Table 4. The energy losses and its deviation percentage in different cases with one DGSC.

\begin{tabular}{cccccccc}
\hline Item & Base Network & Case 1 & Case 2 & Case 3 & Case 4 & Case 5 & Case 6 \\
\hline $\begin{array}{c}\text { Energy losses } \\
\text { value } \\
\begin{array}{c}\text { Maximum } \\
\text { deviation }(\%)\end{array}\end{array}$ & 2023 & 1259.39 & 1267.52 & 1645.61 & 2032.79 & 1285.4 & 1759.1 \\
\hline
\end{tabular}

In this section, the optimal planning of two DGSCs in six different cases is performed using the MRFO method. The optimal generation of DGSCs is shown in Figure 17. The first DGSC is placed at bus 50 with size of $1000 \mathrm{~kW}$ and the second DGSC is located at bus 52 with size of $965 \mathrm{~kW}$ in the network. In allocation of two DGSCs considering smart load case, the energy loss is obtained $771.77 \mathrm{~kW}$, which is reduced by $63.3 \%$ compared to the energy losses with allocation of one DGSC (1259.39 kW). The daily energy losses considering smart load is illustrated without DGSCs and with one and two DGSCs in Figure 18. This figure proved that effective impact of increasing the number of DGSCs in loss of energy reduction.

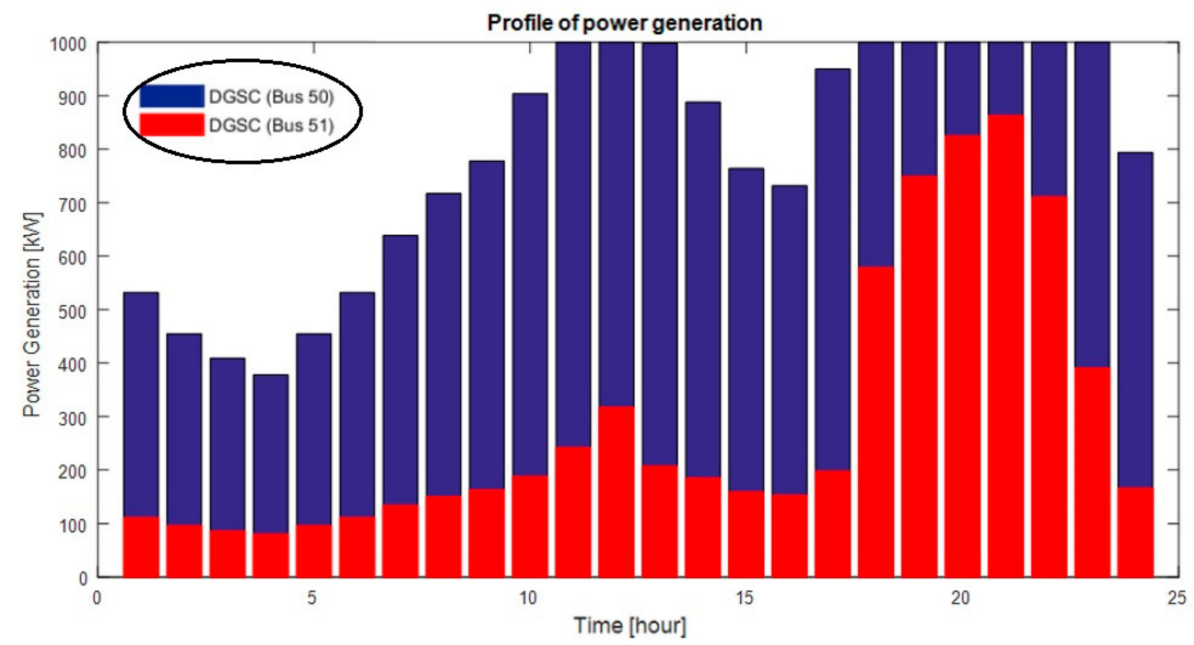

Figure 17. Optimal generation of the DGSCs in smart network. 


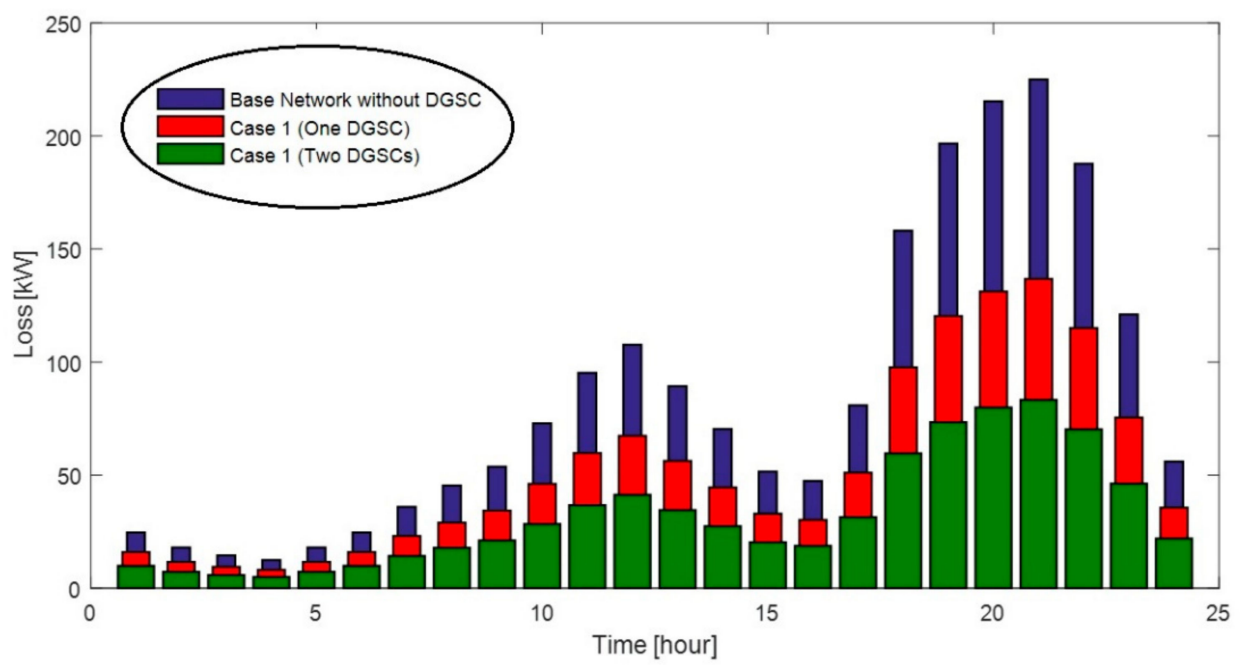

Figure 18. Comparison between hourly energy losses before and after optimal installation of one and two DGSCs in smart network.

In Table 5, loss of energy and the losses reduction percentage of different cases are given. The loss of energy as well as deviation of output power of DGSCs from the optimal value with two DGSCs is presented in Figures 19 and 20. As is clear, the loss of energy during considering smart demand is obtained less than the other cases (estimated demand). Case 2 is closer to the optimal case in view of lower energy losses. The worst case is related to Case 4, where the loss of energy related to conventional demand is more than the value obtained considering the smart demand.

Table 5. The energy losses and its deviation percentage in different cases with two DGSCs.

\begin{tabular}{cccccccc}
\hline Item & Base Network & Case 1 & Case 2 & Case 3 & Case 4 & Case 5 & Case 6 \\
\hline $\begin{array}{c}\text { Energy losses } \\
\text { value } \\
\begin{array}{c}\text { Maximum } \\
\text { deviation of } \\
\text { generation }(\%)\end{array}\end{array}$ & 2023 & 771.77 & 794.37 & 1140.6 & 1387.6 & 819.42 & 1175.4 \\
\hline
\end{tabular}

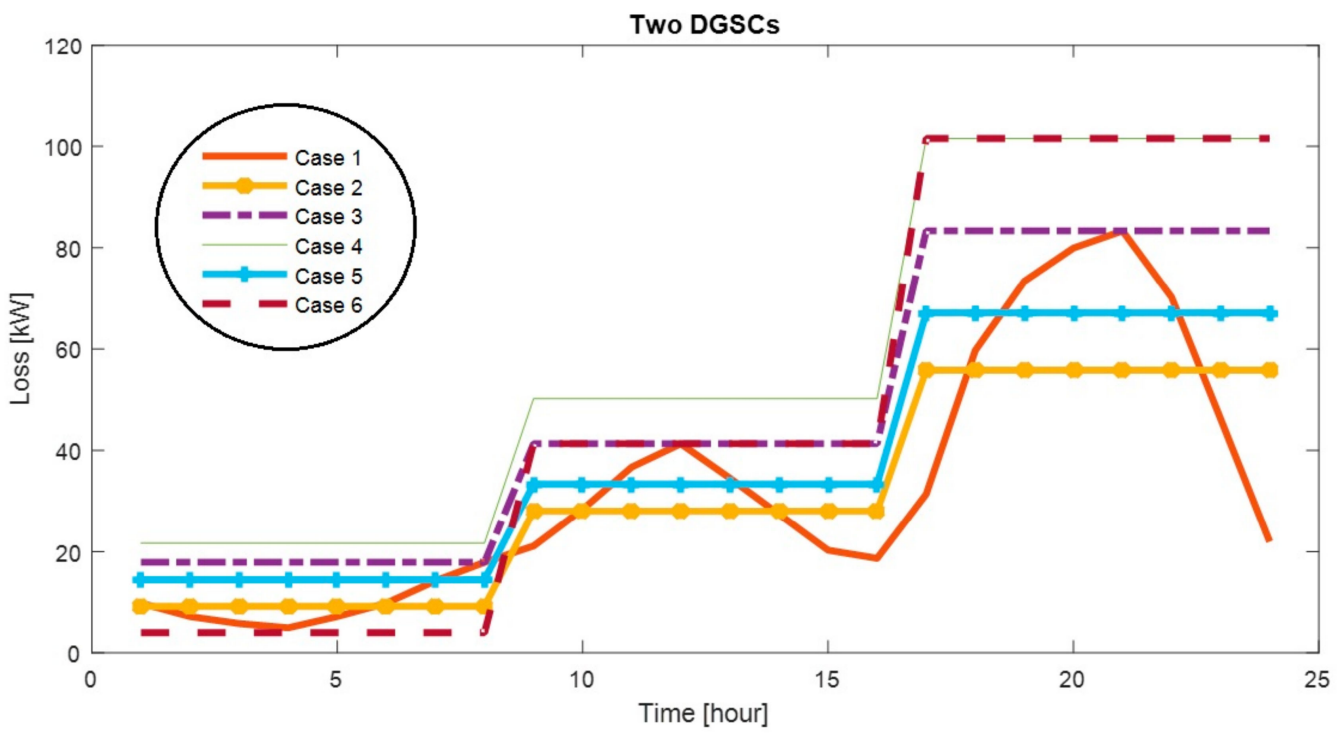

Figure 19. Daily energy loss with two DGSCs for different cases. 


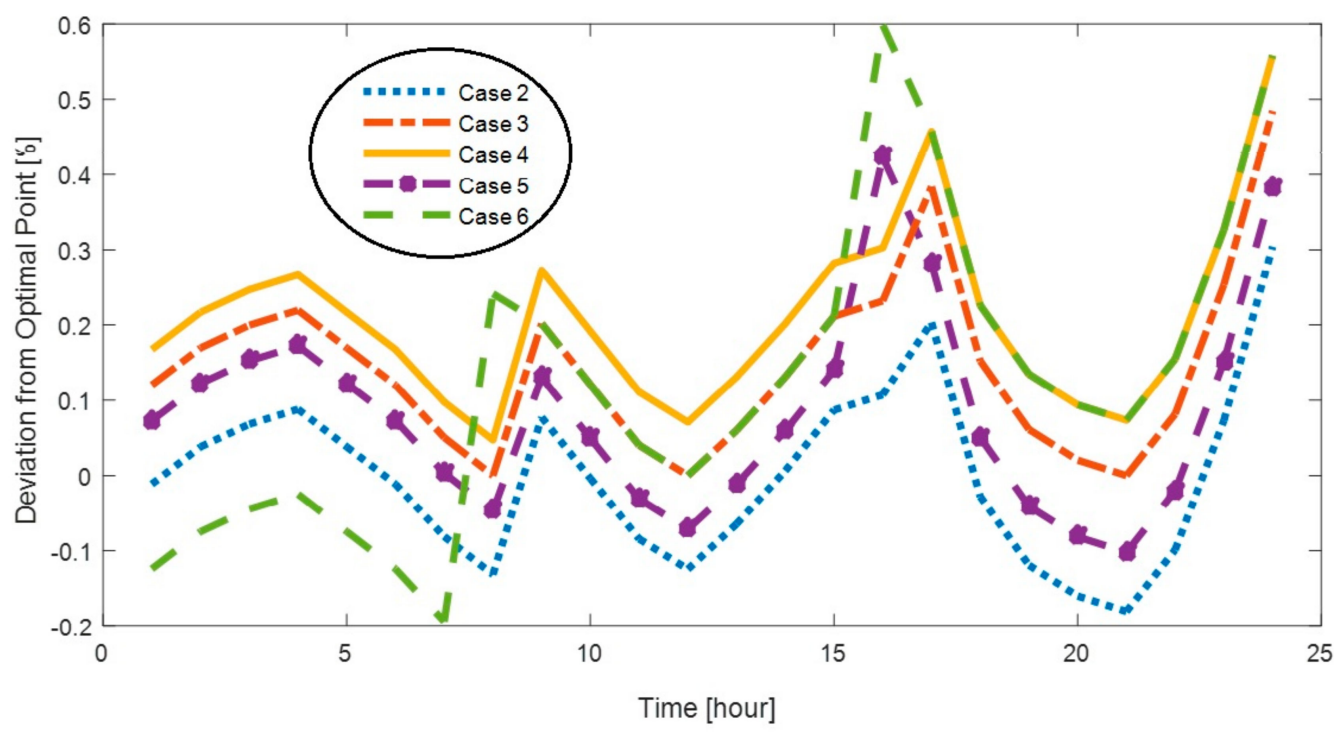

Figure 20. Curve of daily energy losses for different cases with two DGSCs.

\section{Conclusions}

In this study, multi-objective allocation of power generation resources as DGSCs in a smart network is investigated with objective of minimizing the losses and enhancing the voltage profile using the MRFO algorithm. Then, the effect of considering smart loads in planning of DGSCs on the optimal distribution of DGSCs generation is compared to the estimated load in a conventional network with objective of minimizing the loss of energy. The proposed method is implemented on IEEE 69-bus network considering one and two DGSCs using the MRFO. The results obtained from the MRFO are compared with the PSO to evaluate the capability of the MRFO. The results showed that the best solution as minimum loss of energy is obtained considering exact data as smart demand in Case 1. The worst situation occurred in cases 4 and 6 because the estimated loads are higher than the smart demand. The highest difference in the energy losses occurred in Case 4 and the lowest in Case 2, so the results clarified that any error in load forecasting leads to non-optimal operating points and more energy losses. Moreover, the results showed that increasing the DGSC number significantly reduced daily energy losses. In the multi-objective allocation and planning of one and two DGSCs, the computational time obtained is 480 and $570 \mathrm{~s}$.

Access to accurate network load data in a conventional network as well as load uncertainty are the main research limitations. The optimal allocation and planning of power generation resources as distributed generation with scheduling capability with objective of reliability improvement considering load uncertainty is suggested for future work.

Author Contributions: All authors have contributed equally to this work. All authors of this manuscript jointly have conceived the theoretical analysis, methodology, modeling, and obtained the simulation. All authors have read and agreed to the published version of the manuscript.

Funding: This research received no external funding.

Institutional Review Board Statement: Not applicable.

Informed Consent Statement: Not applicable.

Data Availability Statement: Not applicable.

Conflicts of Interest: The authors declare no conflict of interest. 


\section{References}

1. Jafar-Nowdeh, A.; Babanezhad, M.; Arabi-Nowdeh, S.; Naderipour, A.; Kamyab, H.; Abdul-Malek, Z.; Ramachandaramurthy, V.K. Meta-heuristic matrix moth-flame algorithm for optimal reconfiguration of distribution networks and placement of solar and wind renewable sources considering reliability. Environ. Technol. Innov. 2020, 20, 101118. [CrossRef]

2. Moghaddam, M.J.H.; Kalam, A.; Shi, J.; Nowdeh, S.A.; Gandoman, F.H.; Ahmadi, A. A New Model for Reconfiguration and Distributed Generation Allocation in Distribution Network Considering Power Quality Indices and Network Losses. IEEE Syst. J. 2020, 14, 3530-3538. [CrossRef]

3. Augusto, A.A.; de Souza, J.C.S.; Do Coutto Filho, M.B.; de Oliveira Rocha, H.R.; Tafur, J.E.V. Optimized Capacitor Placement Considering Load and Network Variability. J. Control Automat. Electr. Syst. 2020, 31, 1489-1498. [CrossRef]

4. Hayerikhiyavi, M.; Dimitrovski, A. Comprehensive Analysis of Continuously Variable Series Reactor Using GC Framework. arXiv Preprint 2021, arXiv:2103.11136.

5. Naderipour, A.; Abdul-Malek, Z.; Hajivand, M.; Seifabad, Z.M.; Farsi, M.A.; Nowdeh, S.A.; Davoudkhani, I.F. Spotted hyena optimizer algorithm for capacitor allocation in radial distribution system with distributed generation and microgrid operation considering different load types. Sci. Rep. 2021, 11, 1-15. [CrossRef]

6. Nowdeh, S.A.; Davoudkhani, I.F.; Moghaddam, M.H.; Najmi, E.S.; Abdelaziz, A.; Ahmadi, A.; Razavi, S.; Gandoman, F. Fuzzy multi-objective placement of renewable energy sources in distribution system with objective of loss reduction and reliability improvement using a novel hybrid method. Appl. Soft Comput. 2019, 77, 761-779. [CrossRef]

7. Naderipour, A.; Abdul-Malek, Z.; Nowdeh, S.A.; Ramachandaramurthy, V.K.; Kalam, A.; Guerrero, J. Optimal allocation for combined heat and power system with respect to maximum allowable capacity for reduced losses and improved voltage profile and reliability of microgrids considering loading condition. Energy 2020, 196, 117124. [CrossRef]

8. Hadidian-Moghaddam, M.J.; Nowdeh, S.A.; Bigdeli, M.; Azizian, D. A multi-objective optimal sizing and siting of distributed generation using ant lion optimization technique. Ain Shams Eng. J. 2018, 9, 2101-2109. [CrossRef]

9. Jahannoush, M.; Nowdeh, S.A. Optimal designing and management of a stand-alone hybrid energy system using meta-heuristic improved sine-cosine algorithm for Recreational Center, case study for Iran country. Appl. Soft Comput. 2020, 96, 106611. [CrossRef]

10. Nowdeh, S.A.; Ghahnavieh, A.R.; Shojaei, H. Reliable Designing of Stand-alone PV/FC Hybrid System. Majlesi J. Electr. Eng. 2013, 7, 41-47.

11. Rajabighahnavieh, A.; Nowdeh, S.A. Optimal PV-FC hybrid system operation considering reliability. Int. J. Electr. Power Energy Syst. 2014, 60, 325-333. [CrossRef]

12. Naderipour, A.; Abdul-Malek, Z.; Nowdeh, S.A.; Kamyab, H.; Ramtin, A.R.; Shahrokhi, S.; Klemeš, J.J. Comparative evaluation of hybrid photovoltaic, wind, tidal and fuel cell clean system design for different regions with remote application considering cost. J. Clean. Prod. 2021, 283, 124207. [CrossRef]

13. Haggi, H.; Nejad, R.R.; Song, M.; Sun, W. A Review of Smart Grid Restoration to Enhance Cyber-Physical System Resilience. In Proceedings of the 2019 IEEE Innovative Smart Grid Technologies-Asia (ISGT Asia), Chengdu, China, 21-24 May 2019; pp. 4008-4013. [CrossRef]

14. Haggi, H.; Marjani, S.R.; Golkar, M.A. The effect of rescheduling power plants and optimal allocation of STATCOM in order to Improve power system static security using TLBO algorithm. In Proceedings of the 2017 Iranian Conference on Electrical Engineering (ICEE), Tehran, Iran, 2-4 May 2017; pp. 1120-1125. [CrossRef]

15. Kayal, P.; Chanda, C.K. Placement of wind and solar based DGs in distribution system for power loss minimization and voltage stability improvement. Int. J. Electr. Power Energy Syst. 2013, 53, 795-809. [CrossRef]

16. Khatod, D.K.; Pant, V.; Sharma, J. Evolutionary programming based optimal placement of renewable distributed generators. IEEE Trans. Power Syst. 2012, 28, 683-695. [CrossRef]

17. Nadhir, K.; Chabane, D.; Tarek, B. Firefly algorithm based energy loss minimization approach for optimal sizing \& placement of distributed generation. In Proceedings of the 2013 5th International Conference on Modeling, Simulation and Applied Optimization (ICMSAO), Hammamet, Tunisia, 28-30 April 2013; pp. 1-5. [CrossRef]

18. Rajaram, R.; Kumar, K.S.; Rajasekar, N. Power system reconfiguration in a radial distribution network for reducing losses and to improve voltage profile using modified plant growth simulation algorithm with Distributed Generation (DG). Energy Rep. 2015, 1, 116-122. [CrossRef]

19. Nguyen, T.T.; Truong, A.V.; Phung, A.-T. A novel method based on adaptive cuckoo search for optimal network reconfiguration and distributed generation allocation in distribution network. Int. J. Electr. Power Energy Syst. 2016, 78, 801-815. [CrossRef]

20. Khalesi, N.; Rezaei, N.; Haghifam, M.-R. DG allocation with application of dynamic programming for loss reduction and reliability improvement. Int. J. Electr. Power Energy Syst. 2011, 33, 288-295. [CrossRef]

21. Ochoa, L.; Harrison, G. Minimizing Energy Losses: Optimal Accommodation and Smart Operation of Renewable Distributed Generation. IEEE Trans. Power Syst. 2010, 26, 198-205. [CrossRef]

22. Nasri, A.; Hamedani Golshan, M.E.; Mortaza Saghaian Nejad, S. Optimal planning of dispatchable and non-dispatchable distributed generation units for minimizing distribution system's energy loss using particle swarm optimization. Int. Trans. Electr. Energy Syst. 2014, 24, 504-519. [CrossRef]

23. Guo, Y.; Lin, Y.; Sun, M. The impact of integrating distributed generations on the losses in the smart grid. In Proceedings of the Power and Energy Society General Meeting, Detroit, MI, USA, 24-28 July 2011; pp. 1-6. 
24. Rahiminejad, A.; Vahidi, B.; Hejazi, M.; Shahrooyan, S. Optimal scheduling of dispatchable distributed generation in smart environment with the aim of energy loss minimization. Energy 2016, 116, 190-201. [CrossRef]

25. Katic, N.; Marijanovic, V.; Stefani, I. Smart grid solutions in distribution networks cost/benefit analysis. In Proceedings of the Electricity Distribution (CICED), 2010 China International Conference, Nanjing, China, 13-16 September 2010; pp. 1-6.

26. Abaide, A.; Canha, L.; Barin, A.; Cassel, G. Assessment of the smart grids applied in reducing the cost of distribution system losses. In Proceedings of the 2010 7th International Conference on the European Energy Market, Madrid, Spain, 23-25 June 2010; pp. 1-6. [CrossRef]

27. Eajal, A.A.; El-Saadany, E.F.; AlHajri, M.F. Distributed Generation planning in smart distribution grids via a meta-heuristic approach. In Proceedings of the 2014 IEEE 27th Canadian Conference on Electrical and Computer Engineering (CCECE), Toronto, ON, Canada, 5-8 May 2014; pp. 1-6. [CrossRef]

28. Alonso, M.; Amaris, H.; Alvarez-Ortega, C. Integration of renewable energy sources in smart grids by means of evolutionary optimization algorithms. Expert Syst. Appl. 2012, 39, 5513-5522. [CrossRef]

29. Wolpert, D.H.; Macready, W.G. No free lunch theorems for optimization. IEEE Trans. Evol. Comput. 1997, 1, 67-82. [CrossRef]

30. Adam, S.P.; Alexandropoulos, S.-A.N.; Pardalos, P.M.; Vrahatis, M.N. No Free Lunch Theorem: A Review. Approx. Optim. 2019, 57-82. [CrossRef]

31. Zhao, W.; Zhang, Z.; Wang, L. Manta ray foraging optimization: An effective bio-inspired optimizer for engineering applications. Eng. Appl. Artif. Intell. 2020, 87, 103300. [CrossRef]

32. Fathy, A.; Rezk, H.; Yousri, D. A robust global MPPT to mitigate partial shading of triple-junction solar cell-based system using manta ray foraging optimization algorithm. Sol. Energy 2020, 207, 305-316. [CrossRef]

33. Lotfipour, A.; Afrakhte, H. A discrete Teaching-Learning-Based Optimization algorithm to solve distribution system reconfiguration in presence of distributed generation. Int. J. Electr. Power Energy Syst. 2016, 82, 264-273. [CrossRef]

34. Baran, M.; Wu, F. Network reconfiguration in distribution systems for loss reduction and load balancing. IEEE Trans. Power Deliv. 1989, 4, 1401-1407. [CrossRef] 